\title{
MATRIZ DE CONTABILIDAD SOCIAL EXTENDIDA AMBIENTALMENTE PARA ANALISIS ECONOMICO DE LA REGION DEL BIO BIO
}

\author{
ENVIRONMENTALLY EXTENDED SOCIAL ACCOUNTING MATRIX \\ FOR ECONOMIC ANALYSIS OF THE BIO BIO REGION
}

\section{CRISTIAN MARDONES P.* \\ Universidad de Concepción}

\section{JORGE SAAVEDRA E. **}

Universidad de Concepción

\begin{abstract}
We elaborate an Environmentally Extended Social Accounting Matrix for the Bío Bío Region. Initially, we apply the Cross Entropy method for updating a Social Accounting Matrix of this region from 1996 to 2006. This matrix provides the basis for a discussion of multipliers, which aims to identify the productive sectors of the region that would generate the most beneficial impacts on production, equity, poverty, and environmental pollution. The paper results are used to assess the priority sectors selected by the Bio-Bio's Productive Development Agency.
\end{abstract}

Keywords: Social Accounting Matrix, Environment, Economic Development.

JEL Classification: C67, Q56, R58.

\section{Resumen}

Se construye una Matriz de Contabilidad Social Extendida Ambientalmente para la Región del Bío Bío. Para ello aplicamos el método indirecto conocido como Cross Entropy para actualizar una Matriz de Contabilidad Social de esta región desde el año 1996 al 2006. Esta matriz sirve de base para un análisis de multiplicadores, cuyo fin es identificar los sectores productivos

* Profesor Asistente Departamento de Ingeniería Industrial. Facultad de Ingeniería Universidad de Concepción. Dirección: Edmundo Larenas 215, Ciudad Universitaria, Concepción, Chile. Tel.: 56-412203614. E-mail: crismardones@udec.cl

** Programa Magíster en Ingeniería Industrial. Universidad de Concepción. E-mail: jorgsaavedra@udec.cl 
de la región que generarían los impactos más beneficiosos sobre la producción, equidad, pobreza, y contaminación ambiental. Los resultados del presente estudio sirven para evaluar los sectores prioritarios escogidos por la Agencia de Desarrollo Productivo de la región del Bío Bío.

\section{Palabras Clave: Matriz de Contabilidad Social, Medio ambiente, Desarrollo Económico.}

Clasificación JEL: C67, Q56, R58.

\section{INTRODUCCION}

Dado el comportamiento sistémico de una economía, resulta difícil, si no imposible, prever todos los efectos que una medida o política económica específica tendrá en un sistema económico determinado, por lo que se requiere de herramientas formales que sean capaces de representar certeramente la situación base y proyecciones de la situación contrafactual. Dentro de este marco, una de las herramientas existentes son las matrices de contabilidad social (SAM, Social Accounting Matrix), que capturan una imagen desagregada de una economía, representando matricialmente todas las transacciones y transferencias (monetarias) ocurridas en esa economía en un determinado periodo. La creación de estas matrices se le atribuye a Sir Richard Stone por su artículo "Input-Output and the Social Accounts" $(1954)^{1} \mathrm{y}$, principalmente, por su trabajo en la década de 1960 desarrollando SAM para el Reino Unido y otros países desarrollados. Posteriormente, serían Pyatt y Thorbecke (1976) quienes en gran medida formalizaron el concepto de una SAM como herramienta de contabilidad social, describiendo la estructura general de éstas e incluyendo mayores detalles que los utilizados hasta ese entonces en las cuentas asociadas a los factores de producción y hogares. Esta formalización continuó con Pyatt y Roe (1977) y su trabajo describiendo en detalle el desarrollo de una SAM para Sri Lanka. Tras más de cuatro décadas desde su concepción, las SAM han sido desarrolladas para distintos países y regiones, y con distintos propósitos. Entre éstos se encuentran estudios de redistribución del ingreso (Llop y Manresa, 2004), estrategias de crecimiento en países en desarrollo (Vos y Jong, 2003), evaluación de políticas económicas (Ciaschini y Socci, 2007), entre otros. Además se han desarrollado diversas extensiones al modelo tradicional, como el trabajo de Uriel et al. (2005) que considera y cuantifica la producción (no remunerada) de servicios en los hogares, o bien la extensión ambiental de las SAM (Keuning y Timmerman, 1995; Morilla y Llanes, 2004; Xie, 2000). En Chile, existen algunos estudios que abordan la construcción de SAM para el país como García y Castillo (1984), Venegas (1995), y Lagos et al. (2003), elaborando SAM para los años 1977,

1 Debe notarse que el artículo de Stone (1954) debe interpretarse en términos de "Input-Output and the National Accounts", según Vanoli (2005). Con lo cual, la SAM de Stone es en realidad la NAM. 
1986 y 1996, respectivamente. La construcción de SAM regionales, por otro lado, ha sido abordada en los trabajos de Rojas (2009), Mardones (2009) y Casanello y Hanne (2004), para los casos de la Región Metropolitana, Región del Bío Bío y Región de Antofagasta, respectivamente.

En Chile, desde hace algunos años como parte de iniciativas de descentralización, se ha buscado el desarrollo a largo plazo de las regiones del país. Uno de los principales mecanismos es mediante la potenciación de ciertos sectores productivos. Sin embargo, cabe preguntarse si los sectores objeto de estas iniciativas de políticas públicas, realmente son los más beneficiosos para la región desde distintas perspectivas económicas, distributivas y ambientales.

Este trabajo busca identificar a los sectores que tendrían el mayor impacto positivo sobre la región al experimentar un incremento de su demanda final, tal como pudiera ser el surgido, por ejemplo, como respuesta a iniciativas que busquen impulsar el nivel de las exportaciones regionales, demanda final $\mathrm{u}$ otro tipo de políticas de fomento productivo. Para ello, el análisis considera el impacto sobre la producción, pobreza, desigualdad, y sobre el nivel de las emisiones contaminantes que los sectores generarían en el escenario bajo estudio.

Como aplicación concreta, se analiza si la Agencia Regional de Innovación y Desarrollo Productivo Región del Bío Bío (ARIDP BIO BIO), constituida a fines del 2006, ha orientado sus recursos hacia los sectores correctos en el marco de sus estrategias de mejora de la capacidad competitiva regional, en base a los cuatro ámbitos de desarrollo económico planteados en este estudio. Los sectores priorizados en base a un consejo estratégico regional son: 'Alimentos', 'Turismo', 'Pesca', 'ForestalMaderero', 'Metalmecánico', 'Petroquímico-Plástico', 'Educación Superior-Ciencia y Tecnología', 'Transporte' y 'Energía Renovable'.

Como principales resultados a partir de la ESAM para la Región del Bío Bío y del indicador de desarrollo económico propuesto es posible concluir que en la mayoría de los casos los sectores priorizados por la ARIDP BIO BIO se encontrarían dentro del conjunto de sectores más relevantes para el desarrollo de la región. Sin embargo, existen algunos sectores en posiciones destacadas del ranking de desarrollo económico que no fueron priorizados.

\section{LA MATRIZ DE CONTABILIDAD SOCIAL}

Una matriz de contabilidad social o SAM representa matricialmente todas las transacciones y transferencias realizadas en un determinado periodo (generalmente un año) dentro de un sistema económico (generalmente un país) entre todos los agentes económicos de éste (Pyatt y Round, 1985; Reinert y Roland-Holst, 1997). Desde un punto de vista matemático, la SAM es una matriz cuadrada en la que cada cuenta es representada por una fila y una columna. Cada celda de esta matriz muestra el pago desde la cuenta de su columna a la cuenta de su fila. Luego, los ingresos de una cuenta son mostrados a lo largo de su fila y sus gastos a lo largo de su columna. Las SAM operan en un escenario de equilibrio total, lo que implica que los ingresos de cada cuenta equivalen a sus gastos. 
El surgimiento de las SAM se explica básicamente por la necesidad de integrar la cuenta de bienes y servicios, y las cuentas de los sectores institucionales en una misma estructura. En particular relacionando los egresos factoriales de las industrias, con los ingresos factoriales de los sectores o agentes. Y éstos, con los gastos en bienes y servicios. Estas transacciones constituyen el "eslabón perdido" de la matriz de insumo producto "cerrada" por la inclusión de los ingresos y gastos de los sectores institucionales. La SAM busca representar el flujo circular de la renta en una economía, mostrando su paso por las actividades productivas, factores de producción, instituciones, consumo y, eventualmente, de vuelta a la producción.

Además, la SAM fue motivada por la insatisfacción que producían los modelos insumo-producto, con sus exclusivos énfasis en la estructura productiva y crecimiento económico de un país, respectivamente, y por no proporcionar información relativa a la distribución del ingreso con el detalle suficiente para analizar la relación entre crecimiento económico y mejoras en la calidad de vida de la población (Keuning y Ruijter, 1988; Robinson, 1989). Como señala Round (2003): “una de las principales características de las SAM es que los hogares y los grupos de hogares son el centro de estas matrices. Sólo si se incluyen detalles de aspectos distributivos de este sector, esta matriz de contabilidad puede adquirir la etiqueta 'social"'.

A pesar de sus ventajas los análisis a partir de matrices insumo-producto o matrices de contabilidad social tienen varias limitaciones, por ejemplo Rickman (1992) y Gillespie et al. (2001) han mostrado que estos sobrestiman los impactos económicos en la ausencia de excesos de oferta, debido a que utilizan los supuestos de precios fijos y de oferta perfectamente elástica. Otra limitación importante es que carecen de estructura económica respecto a los problemas que enfrentan los agentes como la maximización de utilidad de los hogares y maximización de beneficios de las empresas (Partridge y Rickman, 2010), así como también la ausencia de posibilidades de sustitución. Para superar estas limitaciones han surgido herramientas más sofisticadas llamadas modelos de equilibrio general computable que utilizan para su calibración como información base las matrices de contabilidad social.

En este estudio se construye una SAM de la Región del Bío Bío para el año 2006, donde la elección de este año estuvo sujeta a la disponibilidad de información actualizada.

\section{ACTUALIZACION DE UNA SAM APLICANDO EL METODO CROSS ENTROPY}

La construcción de una SAM de forma directa, es decir, construirla recurriendo a toda la información disponible y recolectando la información faltante, es un proceso exigente que involucra un elevado uso de recursos. Por este motivo, se han desarrollado métodos indirectos de construcción, que acortan y simplifican el desarrollo de estas matrices, al estimarlas por medio de la actualización de una SAM ya existente, utilizando para ello sólo la información actualizada que esté disponible. Actualmente, uno de los métodos más populares para abordar este problema es el Cross Entropy (CE), basado en la teoría de información de Shannon (1948). El método entra en la 
categoría de los métodos de minimización de distancias, más específicamente, distancia entre distribuciones de probabilidades, o entropía. En este caso, la medida de distancia utilizada es la medida de entropía cruzada (cross entropy) desarrollada por Kullback-Leibler (1951). Para la formulación de estos problemas, se define la matriz cuadrada $T$ de transacciones (es decir, la SAM), con celdas $t_{i j}$ que satisfacen:

$$
t_{. j}=\sum_{i} t_{i j}
$$

Sea $A$ una matriz de coeficientes técnicos, obtenida a partir de $T$, y con sus celdas, $a_{i j}$, cumpliendo la relación:

$$
a_{i j}=t_{i j} / t_{. j}
$$

Luego, el método CE busca, a partir de una matriz inicial $A^{0}$, encontrar una nueva matriz $\hat{A}^{1}$ cercana a la inicial, que permita obtener una matriz $\mathrm{T}$ que cumpla con la nueva información disponible, donde los índices, 0 y 1, se refieren a las matrices inicial y final respectivamente, y el símbolo $\left(^{\wedge}\right)$ alude a que se trata de estimaciones de las matrices reales, $T^{l}$ y $A^{l}$, no conocidas. Por la forma en que es abordado el problema, los valores finales son aquéllos que minimizan su distancia (en términos de entropía cruzada) con los valores iniciales, cumpliendo a la vez a todas las restricciones (Aceituno, 2008). La principal característica de este método es su flexibilidad, ya que es capaz de incorporar restricciones de diversa índole, existiendo versiones tanto determinísticas como estocásticas. Estas características, sumada a los resultados de estudios como los de Aceituno (2008) y Robinson et al. (2001) que evalúan la capacidad del método para actualizar matrices similares a las SAM (específicamente, matrices insumo-producto), y generar estimaciones cercanas al de la matriz desactualizada inicial, fueron determinantes para que se optara por aplicar el método CE para construir (estimar) la SAM del año 2006.

Para la aplicación del método se tomó como base el código de programación desarrollado por Robinson y El-Said (2000), adaptándolo al caso particular de este estudio. El enfoque dado por estos autores difiere del tradicional enfoque determinístico, pues éstos agregan un componente estocástico al asociar un error de medición a distintos componentes de la SAM. ${ }^{2}$ Para la obtención de la SAM actualizada esta versión del método emplea fundamentalmente tres elementos. El primero de éstos es una SAM base (SAM desactualizada), que en este estudio correspondió a la SAM regional para el año 1996 desarrollada en Mardones (2009). La función que cumple esta matriz es que su estructura es utilizada como referencia a la hora de encontrar una solución, conservando (dentro de lo posible) el orden de magnitud entre las transacciones y manteniendo nulas aquellas transacciones que originalmente lo eran. Lo anterior es

2 Para un análisis detallado del procedimiento se sugiere revisar "Gams Code For Estimating A Social Accounting Matrix (SAM) Using Cross Entropy (CE) Methods”, Robinson y El-Said (2000). 
realizado considerando simultáneamente la nueva información disponible, especificada en las restricciones definidas. Al segundo elemento se le denomina 'Macro SAM', y consiste en una SAM propiamente tal, pero con un bajo nivel de desagregación en sus cuentas. Por último, el tercer elemento es la SAM inicial actualizada, que corresponde a la SAM desagregada y actualizada con todos los valores conocidos. Tanto el segundo, como el tercero de estos elementos son utilizados para definir las restricciones involucradas en el problema de minimización. Sin embargo, un punto importante es que las restricciones derivadas de éstos forman parte de las restricciones 'potenciales', dependiendo el número real de restricciones a emplear dada la capacidad del programa de encontrar una solución. Así, el proceso de encontrar una solución es un proceso gradual, y de prueba y error, donde mientras el algoritmo converja, paulatinamente se van agregando restricciones y/o probando combinaciones distintas de éstas. En el caso de este estudio, el conjunto de restricciones potenciales surgió tanto de valores conocidos, como estimados, obtenidos siguiendo los procedimientos descritos en el Apéndice $\mathrm{C}$, y que involucraron principalmente el uso de información proveniente de fuentes como el Banco Central, INE, y de la encuesta CASEN 2006, así como de suponer que ciertas relaciones manifestadas en la SAM del '96 se mantenían constante entre ambos periodos.

Para la formulación del problema de minimización definimos los siguientes elementos:

$\bar{A}(i, j)$ : coeficiente técnico de la celda $(i, j)$ en la SAM base.

$A(i, j)$ : coeficiente técnico estimado por el método CE para la celda $(i, j)$.

$\bar{W}(k, w)=\bar{W}=1 / m$ : asumiendo una distribución uniforme para el error de medición, corresponde a la probabilidad a priori de obtener un cierto error de medición en cualquiera de los agregados macro (con $m$ igual al número de elementos del set de error $w)$.

$W(k, w)$ : probabilidad estimada por el método CE para el error asociado al agregado macro $k$-ésimo.

$G^{(k)}$ : corresponde a una matriz $n \times n$ (mismas dimensiones que la SAM) definida para cada una de las restricciones de agregados macro (o incluso celdas individuales), con valor uno para celdas pertenecientes a la restricción y cero en otro caso, $\gamma^{k}$ es el valor de la restricción $k$, mientras que $e_{k}$ es el error asociado a ésta.

$T(i, j)$ : valor de transacción estimado por el método CE para la celda $(i, j)$

Luego, el problema de minimización utilizado en la obtención de la SAM fue el siguiente:

$$
\begin{gathered}
\operatorname{Min}_{\{A, W\}} \text { ENTROPIA }=E=\sum_{i} \sum_{j} A(i, j) \cdot[\ln A(i, j)-\ln \bar{A}(i, j)] \\
+\sum_{k} \sum_{w} W(k, w) \cdot[\ln \mathrm{W}(k, w)-\ln (\bar{W})]
\end{gathered}
$$

s.a.

$$
\sum_{i} \sum_{j} G_{i, j}^{(k)} T_{i, j}=\gamma^{(k)}+e_{k} \quad, \forall k
$$




$$
\begin{array}{cl}
\sum_{i} A(i, j)=1 & , \forall i \\
\sum_{i} \bar{A}(i, j)=1 & , \forall i \\
\sum_{w} W(k, w)=1 & , \forall k \\
0 \leq W(k, w) \leq 1 & , \forall k, w \\
0 \leq A(i, j) \leq 1 & , \forall i, j \\
0 \leq \bar{A}(i, j) \leq 1 & , \forall i, j
\end{array}
$$

Una vez resuelto el problema, el método fue capaz de generar una SAM regional actualizada al 2006. Sin embargo, el desempeño real de esta solución no puede ser evaluado mientras no se conozca la SAM real, pudiendo existir otras combinaciones de restricciones que logren que el método converja a soluciones mejores. Otro punto importante, es que al agregar un componente estocástico a la medición de los elementos de la SAM el método en su búsqueda de una solución altera gran parte de los valores especificados en estas restricciones. Por esta razón, algunos de los valores obtenidos de la aplicación del método CE fueron extraídos de la nueva SAM y reemplazados con valores pertenecientes al conjunto de restricciones potenciales. Tras todo lo anterior, la matriz resultante fue balanceada aplicando una versión del método CE creada con este propósito, lográndose finalmente el balance (ingresos iguales a los gastos) entre todas las cuentas de la SAM.

Si bien el procedimiento anterior logró generar una SAM actualizada al 2006, uno de los propósitos de este estudio era el análisis de tópicos relacionados a la distribución del ingreso, por lo que se requería un mayor nivel de desagregación en las cuentas asociadas al 'Factor Trabajo' y 'Hogares'. A mayor número de cuentas, mayor es el error esperado de los métodos indirectos de construcción, razón por la cual estas cuentas fueron desagregadas sólo una vez estimada la SAM del 2006. Utilizando información derivada de la encuesta CASEN 2006 y de la VI Encuesta de Presupuestos Familiares (EPF), fue posible desagregar estas cuentas según tres niveles de calificación laboral: 'no calificado', 'semicalificado', y 'calificado' (cuenta 'Factor Trabajo'), y según quintiles de ingreso (cuenta 'Hogares'). No obstante, hacer esto produjo un nuevo desbalance, por lo que nuevamente fue implementada la versión de balanceo del método CE. 


\section{EXTENSION AMBIENTAL DE LA SAM}

Un elemento no considerado por las SAM, al menos en su formato tradicional, es que la actividad económica tiene un impacto sobre el medio ambiente. Lo anterior se da tanto por el uso exacerbado de recursos naturales, como por la emisión de contaminantes. Diversos autores han dado cuenta de la importancia de lo anterior, ampliando las SAM para consideraciones medioambientales. Una de estas variantes corresponde a la SAM extendida ambientalmente o ESAM $^{3}$ (Environmentally Extended Social Accounting Matrix), consistente en una matriz de contabilidad social que aparte de contar con las tradicionales cuentas en términos monetarios, incluye cuentas ambientales tanto en términos monetarios como físicos para complementar la SAM y dar cuenta de la relación existente entre una economía y el ambiente. Esta integración entre estadísticas económicas y ambientales fue motivada principalmente por las dudas que generaban los tradicionales indicadores económicos como medidas de desarrollo económico sustentables en el largo plazo. Dado que estos indicadores no consideraban ni los cambios en la calidad del ambiente, ni la disminución de recursos naturales, factores considerados relevantes para el crecimiento económico sostenido y para los incrementos en el bienestar de la población (Bartelmus et al., 1991).

Siendo uno de los objetivos de este estudio el análisis del impacto ambiental generado por las actividades productivas, la SAM anteriormente generada fue extendida ambientalmente, es decir, fue transformada en una ESAM. Al igual que con las SAM, el formato dado a una ESAM va a estar sujeto a los objetivos que se tengan y a la disponibilidad de información. Fue este último punto lo que provocó que en la ESAM construida para este trabajo, la extensión fuese hecha únicamente en base a las emisiones contaminantes generadas por las actividades productivas. Las fuentes de información empleadas para esto correspondieron al Inventario de Emisiones Atmosféricas del Gran Concepción, Inventario de Emisiones de Chillán y Los Angeles (CONAMA BIO BIO), y al Registro de Emisiones y Transferencias de Contaminantes (RETC) ${ }^{4}$ considerando emisiones hechas tanto al aire como al agua. En relación a las emisiones al aire, los contaminantes incorporados fueron: MP10 (material particulado de diámetro menor o igual a 10 micrones), $C O$ (monóxido de carbono), $N O x$ (óxidos de nitrógeno), $\mathrm{COV}$ (compuestos orgánicos volátiles), $\mathrm{SO}$ (óxidos de azufre) y $\mathrm{NH}_{3}$ (amoníaco), mientras que los contaminantes al agua fueron: aceites y grasas, cloruros y sulfatos. La SAM extendida ambientalmente para la Región del Bío Bío con año base 2006 a partir de aquí, se le denominará ESAMR-2006.

\section{ANALISIS EN BASE A MULTIPLICADORES CONTABLES}

El análisis basado en multiplicadores contables reconoce e incorpora la interdependencia existente entre los distintos agentes de una economía, lo que permite dar cuenta del impacto que shocks exógenos tienen sobre los distintos componentes que

\footnotetext{
Nombre propuesto por Xie (1995).

4 Plataforma disponible en la página web: http://www.conama.cl/retc
} 
la conforman. Su uso más común se encuentra en la matriz inversa de Leontief, con la que es posible medir el efecto que tienen variaciones en la demanda final sobre los niveles de producción de la economía en estudio. El procedimiento con que se obtienen estos multiplicadores requiere definir qué cuentas de la SAM serán consideradas endógenas, y cuáles exógenas, con estas últimas agrupadas en una única cuenta en la cual se manifiesta el shock exógeno mencionado anteriormente. Tras esto, se calcula la matriz $A_{S A M}$, obtenida al dividir cada uno de los elementos de las cuentas endógenas de la SAM, por el respectivo total columna de esa cuenta. Luego, descomponiendo el ingreso de las cuentas endógenas, entre el proveniente de las cuentas exógenas y aquél que proviene de las mismas cuentas endógenas, con un poco de álgebra matricial se obtiene la matriz de multiplicadores, $M_{S A M}$, que explica el ingreso total de las cuentas endógenas en términos del ingreso proveniente de su relación con las cuentas exógenas, y donde ésta se define como:

$$
M_{S A M}=\left(I-A_{S A M}\right)^{-1}
$$

con $I$ igual a una matriz identidad de las mismas dimensiones que $A_{S A M} \cdot{ }^{5}$ La matriz inversa de Leontief, citada anteriormente, es sólo un caso particular de la matriz anterior, en la cual sólo las actividades productivas son consideradas endógenas, y donde todos los componentes de la demanda final son definidos exógenamente.

De esta forma, la ESAMR-2006 fue utilizada para generar una matriz $M_{S A M}$, la cual sería la base para el análisis de los impactos generados por los shocks, y que consideró como cuentas endógenas a las asociadas a los hogares, factores de producción, y actividades productivas. Luego, cada una de las celdas de la matriz muestra la variación que sufre el ingreso de las cuentas (endógenas) de las filas, ante variaciones unitarias del ingreso de las cuentas (endógenas) de las columnas, donde esta inyección de ingreso proviene de las cuentas exógenas que en este estudio corresponden a los componentes de la demanda final (excluido el consumo de los hogares, por ser endógeno). Así, el análisis permitió no sólo evaluar cómo variaciones en la demanda final (por ejemplo, de la demanda del resto del país y/o del resto del mundo) de los distintos sectores afectan los niveles de producción de la economía regional (como ocurre con la matriz inversa de Leontief), sino que este análisis pudo extenderse para considerar cómo estas variaciones repercuten en la distribución del ingreso y en los ingresos del quintil más pobre, evaluando de esta manera sus efectos en la pobreza. De igual forma, mediante la utilización de indicadores medioambientales ligados a la emisión de contaminantes por parte de los sectores productivos, pudo analizarse el impacto ambiental generado en ese escenario. Los indicadores empleados, el procedimiento con el que se obtuvieron y la aplicación dada a éstos, son descritos a continuación.

5 Para un análisis más detallado del procedimiento se sugiere revisar Defourny y Thorbecke (1984). 


\subsection{Indicadores de Producción}

Los indicadores empleados para este análisis fueron medidas de los encadenamientos hacia atrás y hacia adelante de los distintos sectores. Si bien, la literatura ofrece distintas alternativas para este propósito, en este caso se utilizaron los indicadores obtenidos directamente a partir de la matriz inversa de Leontief y de la matriz $M_{S A M},{ }^{6}$ utilizando los multiplicadores de producción (multiplicadores $m_{i j}$, donde tanto $i$, como $j$ representan actividades productivas). Los encadenamientos hacia atrás $B L_{j}$ fueron calculados, para cada sector $j$, como la suma a lo largo de las columnas de los multiplicadores productivos.

$$
B L_{j}=\sum_{i} m_{i j}
$$

En este caso, el indicador muestra el efecto agregado sobre la producción de todos los sectores, dado un incremento unitario en la demanda final del sector $j$. Para el caso de los encadenamientos hacia adelante, el indicador $F L_{j}$ se calcula como la suma de los elementos de las filas de la matriz inversa de Leontief o alternativamente la matriz $M_{S A M}$, aunque en este último caso sólo considerando los multiplicadores productivos.

$$
F L_{j}=\sum_{j} m_{i j}
$$

Este indicador mide el encadenamiento hacia delante del sector $i$, al indicar cuánto debería crecer la producción de éste, ante un incremento unitario en la demanda final de todos los sectores. Una vez calculados estos indicadores para cada uno de los sectores, el análisis se basó en identificar a los sectores más relacionados con el resto desde el punto de vista productivo, y observar el grado de concordancia entre éstos y los sectores priorizados por la Agencia Regional de Innovación y Desarrollo Productivo Región del Bío Bío. Para cada tipo de encadenamiento, el criterio utilizado para evaluar el grado de interconectividad de los sectores fue si los indicadores de éstos se encontraban en un nivel mayor o inferior al de la media sectorial o bien estableciendo un ranking que ordenó de mayor a menor ambos tipos de encadenamiento para cada sector.

\subsection{Indicadores de Ingreso}

Este análisis consideró el impacto sobre el ingreso laboral e impacto sobre el ingreso total de los hogares.

En el primer caso la matriz de multiplicadores basado en la $M_{S A M}$ fue utilizada para calcular la distribución porcentual del ingreso laboral generado por cada sector (bajo la situación analizada de incremento en la demanda final), entre los tres niveles

6 Aunque también se reportan los indicadores basados en la Matriz Inversa de Leontief obtenidos con el mismo método que los de la $M_{S A M}$. 
de calificación considerados. Luego, el análisis de la distribución del ingreso laboral estuvo dirigido a identificar los sectores que retribuyeran en mayor y en menor medida a su fuerza laboral según los tres niveles de calificación empleados (no calificado, semicalificado, y calificado).

En el segundo caso se evalúa el impacto que producen variaciones de la demanda final sobre el ingreso total de los hogares, calculando el ingreso total que llega a los hogares y distribuyéndolo por quintiles de ingreso. Otro tipo de indicador utilizado, fue la razón entre los multiplicadores que cada sector presentaba en torno al ingreso recibido por el quintil de mayores ingresos y el de menores ingresos, indicador de desigualdad que fue denominado $q 5 / q 1$. El énfasis estuvo puesto en identificar los sectores que mayor repercusión tienen en la reducción de la pobreza, por lo cual, la discusión se situó en torno a los sectores que mayor ingreso porcentual aportasen al quintil de menores ingresos. En cuanto al uso del indicador $q 5 / q 1$, éste estuvo dirigido a identificar los sectores que mejoraran en mayor medida la distribución del ingreso, y contrastar esta información con la de los sectores que representaran el mayor aporte para combatir la pobreza.

\subsection{Indicadores Ambientales}

En este caso, los indicadores empleados fueron obtenidos de la siguiente manera. En primer lugar, el valor de las emisiones (al aire y agua) de los distintos contaminantes $E_{i l}$, para cada sector fue dividido por la producción bruta del sector correspondiente $y_{i}$, es decir:

$$
E_{i l}^{Y}=E_{i l} / y_{i}
$$

Este indicador muestra cuánto varía el nivel de las emisiones del contaminante $l$ emitido por el sector $i$, por unidad de producción, asumiendo una relación lineal entre el nivel de las emisiones y la producción, y por tanto, sobrevalorando el nivel de las emisiones. Sin embargo, el indicador anterior fue empleado para obtener el verdadero indicador que interesaba, uno que mostrara la variación total en el nivel de emisiones de un cierto contaminante, por unidad de variación en la demanda final de algún sector determinado, considerando a la vez las interrelaciones entre los sectores, es decir, un indicador que considerara los multiplicadores de producción. Para esto, los nuevos indicadores fueron construidos como:

$$
E_{i l}^{D}=\sum_{j} m_{i j} \cdot E_{j l}^{Y}
$$

Así, estos indicadores muestran cuánto varía el nivel de las emisiones del contaminante $l$ (en toneladas), ante un aumento unitario en la demanda final del sector $i$, considerando todos los efectos, directos e indirectos, que esta variación produce en la producción de todos los sectores. De este modo fue posible determinar para cada contaminante, los sectores que en el escenario estudiado (aumento de su demanda 
final) generasen las mayores emisiones totales por efecto directo del propio sector y por efectos indirectos de otros sectores impulsados por el primero.

\section{PRINCIPALES RESULTADOS DE LA ESAMR-2006}

La ESAMR-2006 es presentada en el Apéndice A, aunque dado su gran tamaño con cierto grado de agregación de sectores productivos ( 'Recursos naturales', 'Industria', 'Construcción', 'Comercio' y 'Servicios'), ${ }^{7}$ en el mismo apéndice se explica la nomenclatura utilizada para nombrar las cuentas de esta ESAM. Los flujos monetarios se encuentran expresados en miles de millones de pesos del 2006, mientras que las emisiones se encuentran en toneladas. Como principales resultados destaca la fuerte participación industrial en el PIB, la cual alcanza un 34,6\% (incluyendo la industria alimentaria, maderera, papel, química, no metálica, metálica básica, metalmecánica y resto de industria). Por otro lado, la inversión se estaría concentrando casi en su totalidad en productos asociados a los sectores 'Construcción' (51,2\%) y 'Productos Metálicos, Maquinaria y Equipos' (37,3\%). Conforme a lo estimado en base a la EPF, sólo el quintil de mayores ingresos ahorraría, específicamente, alrededor del 9,8\% de su ingreso total. En cuanto a la distribución del ingreso laboral se obtuvo que el $41,6 \%$ se paga al personal menos calificado, el 30,5\% al personal semicalificado, y el restante $27,8 \%$ al personal calificado.

\section{FIGURA 1}

\section{PARTICIPACION SECTORIAL ESTIMADA EN EL PIB REGIONAL (ESAMR-2006) ${ }^{8}$}

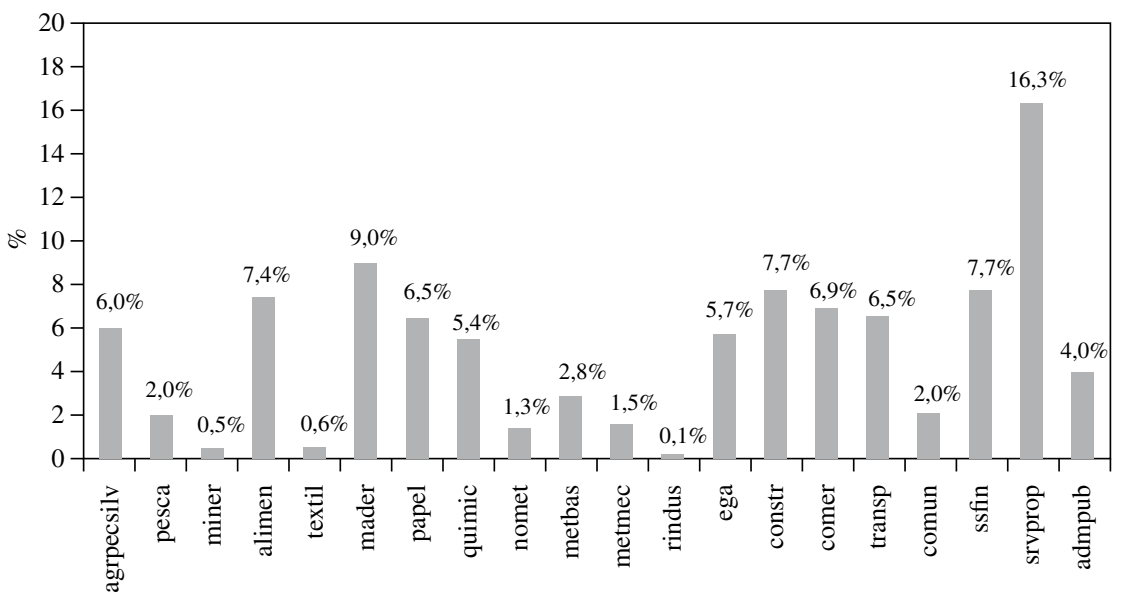

Fuente: Elaboración propia en base a ESAMR-2006.

7 La matriz completa puede ser solicitada a los autores.

8 Para obtener el significado de la abreviatura utilizada para cada sector ver la Tabla A1 del Apéndice A. 
Por otra parte, el ingreso total estaría notoriamente concentrado por el quintil de mayores ingresos con un 44\%, mientras el primer quintil alcanzaría un 8,6\%. Pese a este alto nivel de desigualdad, los resultados al menos darían cuenta que la distribución del ingreso en la Región del Bío Bío sería más equitativa que la distribución a nivel país, pues de la CASEN 2006 se desprende que a nivel nacional el quinto y primer quintil reunirían, respectivamente, el 53,1\% y el 5\% del ingreso total percibido por los hogares. En relación al consumo, éste estaría concentrado principalmente en los bienes y/o servicios asociados a los sectores 'Alimentos, Bebidas y Tabaco' $(15,6 \%)$ y 'Transporte' $(15,4 \%)$, aunque cabe señalar que la estimación a través de los quintiles no es tan acorde a las proporciones de la EPF, diferencia que pudiera atribuirse a que los resultados de las encuestas CASEN y EPF no son del todo combinables, pues el grado de concordancia, entre las razones obtenidas de la SAM y de aquéllas derivadas de la CASEN, fue bastante alto. Por último, en cuanto a la emisión de contaminantes la información recopilada de la CONAMA (ahora Ministerio del Medio Ambiente) muestra que las mayores emisiones a la atmósfera están asociadas a SOx con 19.323,4 t/año, CO con 19.544.5 t/año, NOx con 11.785,2 t/año y MP10 con 7.586 t/año, mientras que las mayores emisiones al agua son $6.886,2$ t/año de sulfatos. Al considerar las emisiones al aire los sectores 'Transporte', 'Electricidad, gas y agua', 'Alimentos, Bebidas y Tabaco', 'Petroquímico-Plástico' generarían la mayor carga al ambiente, representando el $81,1 \%$ del total, mientras que las emisiones al agua son lideradas por el sector 'Papel e Imprentas' con un $89 \%$.

\section{RESULTADOS ANALISIS EN BASE A MULTIPLICADORES CONTABLES}

En esta sección son expuestos los resultados del análisis sobre la base de multiplicadores realizado a partir de la ESAMR-2006. Debido a que la identificación dada por la Agencia Regional de Innovación y Desarrollo Productivo Región del Bío Bío (ARIDP BIO BIO) a los sectores difiere a la utilizada en este estudio, fue necesario establecer las equivalencias entre estas definiciones, para lo cual se incluye Tabla 1.

En el caso del sector prioritario Turismo no fue posible encontrar una equivalencia razonable ya que en la SAM sólo disponemos del sector Comercio, Restaurantes y Hoteles. Para el sector prioritario Educación Superior, Ciencia y Tecnología tampoco existe un sector equivalente razonable en el sector Servicios y Propiedad de Vivienda. Con el sector prioritario 'Forestal-Maderero' la situación fue levemente distinta, el problema radicó en que para representar completamente a este sector se debió asociar dos sectores a él, específicamente los sectores 'A gropecuario-silvícola' y 'Madera y Muebles'. Luego, el análisis en torno a este sector se basó en sus dos sectores asociados, mencionándose, cuando la situación lo amerita, las discrepancias y similitudes surgidas en los resultados de ambos sectores. 
TABLA 1

EQUIVALENCIA SECTORIAL CON LA ARIDP BIO BIO

\begin{tabular}{|ll|}
\hline Sector prioritario & Sector(es) equivalente(s) \\
\hline Alimentos & Alimentos, Bebidas y Tabaco \\
Turismo & Equivalencia No Disponible \\
Pesca & Pesca \\
Forestal-Maderero & Agropecuario-silvícola \\
& Madera y Muebles \\
Metalmecánico & Productos Metálicos, Maquinaria y Equipos \\
Petroquímico-Plástico & Química, Petróleo, Caucho y Plástico \\
Educación Superior-Ciencia y Tecnología & Equivalencia No Disponible \\
Transporte y Logística & Transporte \\
\hline
\end{tabular}

Fuente: Elaboración propia.

\subsection{Análisis de Impactos Productivos}

Como fue mencionado en la quinta sección, el análisis de este tipo de impacto fue obtenido basándose en los encadenamientos productivos que poseen los sectores, donde la magnitud de éstos fue calculada a partir de la matriz de multiplicadores desarrollada.

En la Tabla 2 se observa cuales sectores poseen encadenamientos hacia atrás superiores respecto a la media sectorial (ya sea utilizando para su cálculo la matriz inversa de Leontief o la matriz $M_{S A M}$ ), lo que indica que estos sectores por medio de su demanda en insumos son especialmente capaces de transmitir los beneficios de cualquier incremento en su demanda final al resto de los sectores y, por tanto, iniciativas tendientes a su desarrollo tienen el potencial de tener importantes efectos en la producción regional. Para ilustrar el significado de estos indicadores, tomemos el caso del sector 'Papel e Imprentas', cuyo indicador, al tener un valor de 2,14 o 3,78 según la matriz empleada, indicaría que ante un aumento unitario de la demanda final de este sector, la producción regional (suma de todos los sectores) aumentaría en 2,14 o 3,78 unidades. ${ }^{9}$ Si consideramos la medida más tradicional de encadenamiento hacia atrás basada en la matriz inversa de Leontief y situamos el análisis en torno a los sectores prioritarios, se observa que de los sectores elegidos por la ARIDP BIO BIO, sólo los sectores 'Pesca', 'Petroquímico-Plástico' y 'Metalmecánico' tienen encadenamientos hacia atrás relativamente altos.

Por otro lado, en la Tabla 3 se presenta el análisis en torno a los encadenamientos hacia adelante (ya sea utilizando para su cálculo la matriz inversa de Leontief

9 Valor que incluye la unidad inicial que surgió de este sector para satisfacer el incremento que experimentó en su demanda final, así como las unidades adicionales que debió generar producto de los efectos totales que se dieron en el aparato productivo. 


\section{TABLA 2}

ENCADENAMIENTOS HACIA ATRAS Y MEDIA SECTORIAL

\begin{tabular}{|lccc|}
\hline \multicolumn{1}{|c}{ Sector } & $\begin{array}{c}\text { Matriz } \\
\text { Inversa de } \\
\text { Leontief }\end{array}$ & $\begin{array}{c}\text { Matriz } \\
\text { Inversa de } \\
\text { SAM }\end{array}$ & $\begin{array}{c}\text { Sector } \\
\text { prioritario }\end{array}$ \\
\hline agrpecsilv & 2,12 & 3,81 & SI \\
pesca & 3,22 & 4,13 & SI \\
miner & 2,79 & 1,60 & NO \\
alimen & 2,03 & 4,22 & SI \\
textil & 1,00 & 1,85 & NO \\
mader & 2,04 & 4,85 & SI \\
papel & 2,14 & 3,78 & NO \\
quimic & 2,73 & 4,20 & SI \\
nomet & 2,59 & 2,10 & NO \\
metbas & 2,47 & 2,81 & NO \\
metmec & 2,42 & 2,21 & SI \\
rindus & 3,00 & 1,35 & NO \\
ega & 1,92 & 3,03 & NO \\
constr & 2,23 & 5,47 & NO \\
comer & 2,54 & 6,82 & - \\
transp & 2,29 & 4,13 & SI \\
comun & 2,25 & 2,30 & NO \\
ssfin & 2,01 & 4,78 & NO \\
srvprop & 1,59 & 5,91 & NO \\
admpub & 2,04 & 3,34 & \\
Media & & & \\
\hline
\end{tabular}

Fuente: Elaboración propia.

o la matriz $\left.M_{S A M}\right)$. La producción de estos sectores es especialmente sensible a los cambios que experimente la economía en su conjunto, así como especialmente capaz de estimular la producción de otros sectores por medio de su capacidad de oferta. Tomando el caso del sector 'Petroquímico-Plástico', el valor de 3,13 o 3,16 de su indicador (según la matriz empleada) estaría indicando que si cada sector de la región experimentara un incremento unitario de su demanda final, la producción total de este sector aumentaría en 3,13 o 3,16 unidades. ${ }^{10} \mathrm{Al}$ evaluar el grado de concordancia entre los sectores prioritarios y aquéllos con encadenamientos hacia adelante superiores a la

10 Es decir, que aparte de la unidad producida para satisfacer el incremento unitario de la demanda final que experimentaron todos los sectores, el sector 'Petroquímico-Plástico' produciría 3,13 o 3,16 unidades adicionales producto de los efectos directos e indirectos generados. 
media considerando la medida más tradicional de encadenamiento basada en la matriz inversa de Leontief, se observa que sólo algunos sectores priorizados coinciden con este criterio. El caso contrario ocurre con los sectores 'Pesca', 'Forestal-Maderero' (recordemos que el 'Forestal' está asociado al sector 'Agropecuario-silvícola') y 'Alimentos' los cuales se encuentran bajo la media.

TABLA 3

ENCADENAMIENTOS HACIA ADELANTE Y MEDIA SECTORIAL

\begin{tabular}{|lccc|}
\hline Sector & $\begin{array}{c}\text { Matriz Inversa de } \\
\text { Leontief }\end{array}$ & $\begin{array}{c}\text { Matriz Inversa } \\
\text { de SAM }\end{array}$ & Sector prioritario \\
\hline agrpecsilv & 2,25 & 4,13 & SI \\
pesca & 2,17 & 3,28 & SI \\
miner & 3,03 & 4,16 & NO \\
alimen & 1,77 & 3,92 & SI \\
textil & 1,65 & 4,91 & NO \\
mader & 1,66 & 1,76 & SI \\
papel & 1,82 & 1,99 & NO \\
quimic & 3,13 & 3,16 & SI \\
nomet & 1,94 & 3,52 & NO \\
metbas & 2,82 & 3,07 & SI \\
metmec & 3,17 & 3,28 & NO \\
rindus & 1,60 & 4,45 & NO \\
ega & 2,18 & 4,50 & NO \\
constr & 2,32 & 2,25 & - \\
comer & 2,92 & 2,71 & SI \\
transp & 2,44 & 5,14 & NO \\
comun & 1,84 & 5,50 & NO \\
ssfin & 3,59 & 4,70 & - \\
srvprop & 1,87 & 3,46 & NO \\
admpub & 1,25 & 1,79 & \\
& & & \\
Media & 2,27 & 3,58 & \\
\hline
\end{tabular}

Fuente: Elaboración propia.

\subsection{Análisis de Impactos Distributivos y Sobre la Pobreza}

Los resultados en torno a la distribución del ingreso laboral se muestran en la Tabla 4, de la cual se desprende que en el escenario considerado de aumento de la demanda final, los sectores 'Pesca', 'Agropecuario-silvícola' y 'Construcción' son aquellos en los que la mayor parte del ingreso laboral fluye hacia el personal no calificado, en general los sectores industriales entregan la mayor parte del ingreso a la mano de obra semicalificada, mientras que los sectores asociados a 'Servicios Financieros y Empresariales', 'Servicios Personales y Propiedad de Vivienda' y 'Administración Pública' son aquellos en los cuales la mano de obra calificada es la que percibiría la mayor parte del incremento. 


\section{TABLA 4}

DISTRIBUCION DEL INGRESO LABORAL POR SECTOR Y NIVEL DE CALIFICACION

\begin{tabular}{|l|c|c|c|c|c|}
\hline \multicolumn{1}{|c|}{ Sector } & nocalif & semicalif & calif & total & $\begin{array}{c}\text { Sector } \\
\text { prioritario }\end{array}$ \\
\hline agrpecsilv & $49,1 \%$ & $27,7 \%$ & $23,2 \%$ & $100 \%$ & SI \\
pesca & $47,1 \%$ & $29,1 \%$ & $23,8 \%$ & $100 \%$ & SI \\
miner & $37,6 \%$ & $33,7 \%$ & $28,6 \%$ & $100 \%$ & NO \\
alimen & $36,3 \%$ & $36,0 \%$ & $27,8 \%$ & $100 \%$ & SI \\
textil & $33,4 \%$ & $35,9 \%$ & $30,7 \%$ & $100 \%$ & NO \\
mader & $34,6 \%$ & $36,6 \%$ & $28,8 \%$ & $100 \%$ & SI \\
papel & $34,2 \%$ & $36,3 \%$ & $29,5 \%$ & $100 \%$ & NO \\
quimic & $33,8 \%$ & $36,9 \%$ & $29,3 \%$ & $100 \%$ & SI \\
nomet & $33,4 \%$ & $36,7 \%$ & $29,9 \%$ & $100 \%$ & NO \\
metbas & $32,5 \%$ & $37,4 \%$ & $30,1 \%$ & $100 \%$ & NO \\
metmec & $33,2 \%$ & $37,4 \%$ & $29,4 \%$ & $100 \%$ & SI \\
rindus & $30,6 \%$ & $36,0 \%$ & $33,4 \%$ & $100 \%$ & NO \\
ega & $28,4 \%$ & $38,2 \%$ & $33,4 \%$ & $100 \%$ & NO \\
constr & $42,9 \%$ & $32,6 \%$ & $24,5 \%$ & $100 \%$ & NO \\
comer & $35,1 \%$ & $36,1 \%$ & $28,8 \%$ & $100 \%$ & - \\
transp & $36,9 \%$ & $37,2 \%$ & $25,9 \%$ & $100 \%$ & SI \\
comun & $35,0 \%$ & $36,1 \%$ & $28,9 \%$ & $100 \%$ & NO \\
ssfin & $19,8 \%$ & $32,5 \%$ & $47,7 \%$ & $100 \%$ & NO \\
srvprop & $23,9 \%$ & $28,2 \%$ & $47,9 \%$ & $100 \%$ & - \\
admpub & $23,3 \%$ & $27,9 \%$ & $48,8 \%$ & $100 \%$ & NO \\
Media & $34,0 \%$ & $34,4 \%$ & $31,5 \%$ & & \\
\hline
\end{tabular}

Fuente: Elaboración propia.

Los resultados sobre la distribución del ingreso de los hogares son mostrados en las Tablas 5 y 6 . Específicamente la Tabla 5 muestra cómo es distribuido el ingreso generado por cada sector entre los quintiles, donde se aprecia que para todos los quintiles, la proporción de ingreso (generado ante un incremento de la demanda final) que cada sector entrega a éstos presenta diferencias relativamente pequeñas, tendiendo a no superar los 5,6 puntos porcentuales en el quintil 1, los 3,3 puntos porcentuales en el quintil $2, \operatorname{los} 1,2$ puntos porcentuales en el quintil $3, \operatorname{los} 3,2$ puntos porcentuales en el quintil 4 y los 7 puntos porcentuales en el quintil 5. Cabe notar que las diferencias en valor absoluto de cada sector sobre el ingreso total distribuido a los hogares son bastante heterogéneas. Destacan los sectores 'Comercio', 'Servicios Personales y Propiedad de Vivienda' y 'Construcción' los que significativamente más le aportarían al ingreso total de los hogares. 
TABLA 5

DISTRIBUCION DE INGRESO APORTADO POR LAS ACTIVIDADES A LOS HOGARES SEGUN SECTOR Y QUINTIL DE INGRESOS

\begin{tabular}{|c|c|c|c|c|c|c|c|}
\hline \multirow{2}{*}{ Sector } & \multicolumn{5}{|c|}{ Distribución sectorial por quintil } & \multirow{2}{*}{$\begin{array}{c}\text { Ingreso } \\
\text { total }\end{array}$} & \multirow{2}{*}{$\begin{array}{c}\text { Sector } \\
\text { prioritario }\end{array}$} \\
\hline & $\mathrm{q} 1$ & $\mathrm{q} 2$ & $\mathrm{q} 3$ & $\mathrm{q} 4$ & q5 & & \\
\hline agrpecsilv & $22,2 \%$ & $21,2 \%$ & $20,1 \%$ & $19,3 \%$ & $17,2 \%$ & 0,69 & SI \\
\hline pesca & $22,4 \%$ & $21,4 \%$ & $20,1 \%$ & $19,0 \%$ & $17,1 \%$ & 0,53 & SI \\
\hline miner & $21,4 \%$ & $21,2 \%$ & $20,0 \%$ & $19,1 \%$ & $18,3 \%$ & 0,11 & NO \\
\hline alimen & $19,1 \%$ & $19,6 \%$ & $20,4 \%$ & $21,0 \%$ & $19,9 \%$ & 0,73 & SI \\
\hline textil & $19,7 \%$ & $20,2 \%$ & $20,2 \%$ & $20,1 \%$ & $19,7 \%$ & 0,14 & NO \\
\hline mader & $19,1 \%$ & $19,6 \%$ & $20,4 \%$ & $20,9 \%$ & $20,0 \%$ & 0,89 & SI \\
\hline papel & $18,3 \%$ & $19,0 \%$ & $20,5 \%$ & $21,5 \%$ & $20,7 \%$ & 0,62 & NO \\
\hline quimic & $19,1 \%$ & $19,7 \%$ & $20,4 \%$ & $20,7 \%$ & $20,0 \%$ & 0,57 & SI \\
\hline nomet & $19,6 \%$ & $20,1 \%$ & $20,2 \%$ & $20,2 \%$ & $19,7 \%$ & 0,21 & NO \\
\hline metbas & $19,7 \%$ & $20,2 \%$ & $20,2 \%$ & $20,1 \%$ & $19,7 \%$ & 0,39 & NO \\
\hline metmec & $20,3 \%$ & $20,7 \%$ & $20,2 \%$ & $19,7 \%$ & $19,2 \%$ & 0,26 & SI \\
\hline rindus & $19,3 \%$ & $20,0 \%$ & $20,1 \%$ & $20,2 \%$ & $20,4 \%$ & 0,06 & NO \\
\hline ega & $17,0 \%$ & $18,4 \%$ & $20,5 \%$ & $22,0 \%$ & $22,0 \%$ & 0,45 & NO \\
\hline constr & $22,3 \%$ & $21,6 \%$ & $20,1 \%$ & $18,8 \%$ & $17,2 \%$ & 1,06 & NO \\
\hline comer & $20,7 \%$ & $20,9 \%$ & $20,1 \%$ & $19,5 \%$ & $18,8 \%$ & 1,28 & - \\
\hline transp & $20,4 \%$ & $20,6 \%$ & $20,3 \%$ & $20,0 \%$ & $18,8 \%$ & 0,74 & SI \\
\hline comun & $20,2 \%$ & $20,5 \%$ & $20,2 \%$ & $19,9 \%$ & $19,2 \%$ & 0,28 & NO \\
\hline ssfin & $16,9 \%$ & $18,9 \%$ & $19,7 \%$ & $20,4 \%$ & $24,1 \%$ & 0,89 & NO \\
\hline srvprop & $16,8 \%$ & $18,3 \%$ & $19,8 \%$ & $21,1 \%$ & $24,0 \%$ & 1,26 & - \\
\hline admpub & $18,6 \%$ & $19,8 \%$ & $19,3 \%$ & $19,2 \%$ & $23,2 \%$ & 0,58 & NO \\
\hline
\end{tabular}

Fuente: Elaboración propia.

Al centrarse en los sectores cuyo aporte al quintil de menores ingresos y compararlos con los sectores prioritarios concluimos que la ARIDP BIO BIO habría hecho una elección poco apropiada de los sectores a priorizar para hacer frente a la pobreza, ya que el promedio del impacto de los sectores prioritarios es sólo 0,13 (ante el incremento unitario en la demanda final de cada sector), mientras los sectores 'Comercio', 'Servicios Personales y Propiedad de Vivienda' y 'Construcción' son los que significativamente más le aportarían al ingreso del quintil más pobre con un impacto de 1,28, 1,26 y 1,06, respectivamente.

Por último, en la Tabla 6 se presentan los multiplicadores de ingreso obtenidos para los quintiles de menor y mayor ingreso para cada sector productivo (columnas 2 y 3). Al igual que el resto de los multiplicadores utilizados, éstos dan cuenta de cuánto varía el ingreso de la cuenta endógena analizada (quintiles 1 y 5 en este caso), ante una variación unitaria de la demanda final del sector que corresponda. 
Así, el valor de 0,1 que tiene el multiplicador del quinto quintil para el sector 'Electricidad, Gas y Agua' (fila ega y columna $q 5$ de la Tabla 6), estaría indicando que frente a un aumento de una unidad en la demanda final de este sector, el ingreso del quintil de mayores ingresos aumentaría en 0,1 unidades.

\section{TABLA 6}

MULTIPLICADOR DE INGRESO PARA QUINTILES 1 Y 5

E INDICADOR DE DESIGUALDAD

\begin{tabular}{|c|c|c|c|c|}
\hline \multirow{2}{*}{ Sector } & \multicolumn{2}{|c|}{ Ingreso } & \multirow{2}{*}{$\mathrm{q} 5 / \mathrm{q} 1$} & \multirow{2}{*}{$\begin{array}{c}\text { Sector } \\
\text { prioritario }\end{array}$} \\
\hline & $\mathrm{q} 1$ & q5 & & \\
\hline agrpecsilv & 0,15 & 0,12 & 0,78 & SI \\
\hline pesca & 0,12 & 0,09 & 0,76 & SI \\
\hline miner & 0,02 & 0,02 & 0,86 & $\mathrm{NO}$ \\
\hline alimen & 0,14 & 0,15 & 1,04 & SI \\
\hline textil & 0,03 & 0,03 & 1,00 & $\mathrm{NO}$ \\
\hline mader & 0,17 & 0,18 & 1,05 & SI \\
\hline papel & 0,11 & 0,13 & 1,14 & $\mathrm{NO}$ \\
\hline quimic & 0,11 & 0,11 & 1,05 & SI \\
\hline nomet & 0,04 & 0,04 & 1,00 & NO \\
\hline metbas & 0,08 & 0,08 & 1,00 & $\mathrm{NO}$ \\
\hline metmec & 0,05 & 0,05 & 0,94 & SI \\
\hline rindus & 0,01 & 0,01 & 1,06 & NO \\
\hline ega & 0,08 & 0,10 & 1,30 & NO \\
\hline constr & 0,24 & 0,18 & 0,77 & NO \\
\hline comer & 0,27 & 0,24 & 0,91 & - \\
\hline transp & 0,15 & 0,14 & 0,92 & SI \\
\hline comun & 0,06 & 0,05 & 0,95 & NO \\
\hline ssfin & 0,15 & 0,22 & 1,43 & $\mathrm{NO}$ \\
\hline srvprop & 0,21 & 0,30 & 1,43 & - \\
\hline admpub & 0,11 & 0,13 & 1,25 & $\mathrm{NO}$ \\
\hline
\end{tabular}

Fuente: Elaboración propia.

También se incluye la razón de los multiplicadores del quinto y primer q5/q1 (columna 4), el cual fue utilizado para identificar los sectores que tendrían el mayor impacto en mejorar la distribución del ingreso. Se concluye que los tres sectores cuya estimulación más ayuda a mejorar la equidad son la 'Pesca', 'Construcción', y el 'Agropecuariosilvícola'. También podemos notar que la elección de la ARIDP BIO BIO por priorizar los sectores de 'Alimentos' y 'Petroquímico-Plástico'empeora marginalmente la distribución del ingreso (el 'Madero-Forestal' produce una mejora marginal). 


\subsection{Análisis de Impactos Ambientales}

El último de los análisis efectuados correspondió a la evaluación de los impactos ambientales que los distintos sectores generan en el escenario estudiado de incremento de su demanda final, utilizando para ello los indicadores $E^{D}$ definidos en la sexta sección. El valor de estos indicadores es mostrado en la Tabla 7, separando aquéllos ligados a las emisiones al aire (columnas 3 a la 8) y al agua (columnas 9 a la 11). Por el bajo valor de este indicador, se optó, para efectos de presentación y análisis, considerar el escenario donde la demanda final de los sectores experimenta un aumento de mil millones de unidades. Luego, en forma casi análoga al de los multiplicadores anteriormente empleados (éstos bien podrían ser denominados multiplicadores

TABLA 7

INDICADORES AMBIENTALES DE EMISIONES POR SECTOR Y CONTAMINANTE

\begin{tabular}{|c|c|c|c|c|c|c|c|c|c|c|}
\hline \multirow[b]{2}{*}{ Sector } & \multicolumn{6}{|c|}{ Contaminantes del aire } & \multicolumn{3}{|c|}{ Contaminantes del agua } & \multirow{2}{*}{$\begin{array}{c}\text { Sector } \\
\text { priori- } \\
\text { tario }\end{array}$} \\
\hline & MP10 & $\mathrm{CO}$ & NOx & $\mathrm{COV}$ & SOx & NH3 & $\begin{array}{c}\text { Aceites } \\
\text { y } \\
\text { Grasas }\end{array}$ & Cloruros & Sulfatos & \\
\hline agrpecsilv & 1,00 & 2,56 & 1,93 & 0,40 & 2,3 & 0,13 & 0,07 & 0,09 & 0,26 & SI \\
\hline pesca & 1,23 & 2,25 & 1,74 & 0,35 & 2,1 & 0,13 & 0,07 & 0,09 & 0,28 & SI \\
\hline miner & 0,30 & 0,58 & 0,50 & 0,08 & 0,5 & 0,04 & 0,02 & 0,03 & 0,09 & NO \\
\hline alimen & 1,85 & 3,58 & 4,25 & 0,60 & 7,8 & 0,28 & 0,29 & 0,26 & 0,43 & SI \\
\hline textil & 0,46 & 0,86 & 0,53 & 0,11 & 0,7 & 0,07 & 0,03 & 0,04 & 0,16 & NO \\
\hline mader & 1,56 & 4,74 & 3,43 & 1,05 & 3,1 & 0,20 & 0,11 & 0,14 & 0,61 & SI \\
\hline papel & 2,12 & 6,58 & 2,75 & 0,62 & 3,6 & 0,78 & 0,14 & 2,56 & 8,90 & NO \\
\hline quimic & 1,51 & 2,55 & 1,92 & 1,00 & 7,8 & 0,14 & 0,06 & 0,10 & 0,39 & SI \\
\hline nomet & 2,51 & 1,60 & 10,27 & 0,29 & 1,7 & 0,27 & 0,03 & 0,04 & 0,13 & NO \\
\hline metbas & 8,20 & 1,66 & 1,31 & 0,24 & 1,5 & 0,11 & 0,05 & 0,06 & 0,20 & NO \\
\hline metmec & 1,06 & 1,12 & 0,95 & 0,16 & 0,9 & 0,06 & 0,61 & 0,06 & 0,15 & SI \\
\hline rindus & 0,24 & 0,29 & 0,35 & 0,04 & 0,3 & 0,02 & 0,02 & 0,01 & 0,03 & NO \\
\hline ega & 3,86 & 3,84 & 3,04 & 0,28 & 10,0 & 0,97 & 0,05 & 0,07 & 0,21 & NO \\
\hline constr & 3,29 & 3,75 & 5,32 & 0,58 & 3,3 & 0,25 & 0,11 & 0,12 & 0,38 & NO \\
\hline comer & 2,66 & 8,49 & 5,35 & 1,21 & 5,4 & 0,34 & 0,16 & 0,24 & 0,76 & - \\
\hline $\operatorname{transp}$ & 1,62 & 22,95 & 10,45 & 3,23 & 6,8 & 0,21 & 0,08 & 0,10 & 0,34 & SI \\
\hline comun & 0,52 & 1,15 & 0,94 & 0,16 & 0,9 & 0,07 & 0,03 & 0,05 & 0,17 & NO \\
\hline ssfin & 1,33 & 3,81 & 2,57 & 0,54 & 2,8 & 0,20 & 0,09 & 0,22 & 0,72 & NO \\
\hline srvprop & 1,60 & 4,50 & 3,17 & 0,65 & 3,5 & 0,23 & 0,13 & 0,19 & 0,53 & - \\
\hline admpub & 0,82 & 2,11 & 1,56 & 0,30 & 1,7 & 0,12 & 0,05 & 0,08 & 0,24 & NO \\
\hline Media & 1,89 & 3,95 & 3,12 & 0,60 & 3,33 & 0,23 & 0,11 & 0,23 & 0,75 & \\
\hline
\end{tabular}

Fuente: Elaboración propia. 
ambientales), el valor de cada uno de ellos da cuenta de las toneladas adicionales de contaminante emitido, generadas en conjunto por las actividades productivas, ante un incremento de mil millones de unidades de la demanda final del sector analizado. Así, el valor de 1,62 del indicador de la celda (transp, MP10) indica que si el sector 'Transporte' experimenta un aumento en su demanda final de mil millones de unidades, los efectos, directos e indirectos, que esto produciría en todos los sectores productivos se traducirían en un aumento de 1,62 toneladas en las emisiones de MP10.

Los sectores que producirían el mayor impacto en las emisiones al ambiente son: 'Papel e Imprentas', 'Metálica Básica', 'Electricidad, Gas y Agua' y 'Transporte. Por otra parte, al buscar los valores más altos del indicador para cada contaminante, se determina que el asociado a la emisión de $\mathrm{NOx}$ (óxidos de nitrógeno) del sector 'Transporte' es 10,45; de MP10 del sector 'Metálica Básica' es 8,2, de CO del sector 'Transporte' es 22,95, de SOx (óxidos de azufre) del sector 'Electricidad, Gas y Agua' es 10, de COV del sector 'Transporte' es 3,23, de NH3 del sector 'Electricidad, Gas y Agua' es 0,97, de Aceites y Grasas del sector 'Metalmecánica' es 0,61, de Cloruros y Sulfatos del sector 'Papel e Imprentas' es 2,56 y 8,9, respectivamente. Por último, al evaluar en base a los sectores priorizados por la Agencia Regional de Innovación y Desarrollo Productivo, se obtiene que algunos de ellos tienen ante un incremento de su demanda final un fuerte impacto en la emisión de al menos uno de los contaminantes.

\section{EJEMPLO DE EVALUACION POLITICA DE DESARROLLO ECONOMICO REGIONAL}

Los análisis de la sección anterior permitieron ordenar los sectores según cada uno de los criterios de desarrollo económico considerados. Qué ponderación dar a cada criterio, dependerá finalmente de los objetivos de política y/o intereses del evaluador. Para facilitar y generalizar el análisis, como ejemplo se asumirá que cada criterio tiene igual ponderación y que lo único que interesa es la posición relativa de cada sector dentro de un ranking sectorial por tipo de impacto, y no el valor de los indicadores. De igual forma, la evaluación es efectuada omitiendo el tamaño de los sectores en la economía regional. Los resultados de este ranking son expuestos en la Tabla 8. Si un sector ocupa la primera posición en los encadenamientos productivos hacia atrás y adelante, asociados a la columna "Producción", significa que posee el nivel más alto de encadenamientos hacia atrás y adelante de todos los sectores. La primera posición en la columna "Pobreza" indica que ése sería el sector que al experimentar un aumento en su demanda final terminaría retribuyendo al quintil más pobre en la proporción más alta. Con la columna "Equidad" la primera posición daría cuenta que el sector en cuestión sería el que más mejoraría la distribución del ingreso en el escenario considerado de incremento de su demanda final. Por último, que un sector ocupe la primera posición en la columna "Ambiente" quiere decir que este sector es el que menos emisiones totales de contaminantes (al aire y al agua) genera (directa e indirectamente) al experimentar un incremento de su demanda final. 
TABLA 8

RANKING SECTORIAL SEGUN LOS CUATRO TOPICOS ANALIZADOS Y RANKING GLOBAL

\begin{tabular}{|c|c|c|c|c|c|c|c|}
\hline \multirow{3}{*}{ Sector } & \multirow{3}{*}{$\begin{array}{l}\text { Ligado } \\
\text { al sector } \\
\text { prioritario }\end{array}$} & \multicolumn{5}{|c|}{ Posición en ranking por tipo de impacto } & \multirow{3}{*}{$\begin{array}{c}\text { Ranking } \\
\text { Global }\end{array}$} \\
\hline & & \multicolumn{2}{|c|}{ Producción } & \multirow[b]{2}{*}{ Pobreza } & \multirow[b]{2}{*}{ Equidad } & \multirow[b]{2}{*}{ Ambiente } & \\
\hline & & $\begin{array}{l}\text { Enc. } \\
\text { hacia } \\
\text { atrás }\end{array}$ & $\begin{array}{c}\text { Enc. } \\
\text { hacia } \\
\text { adelante }\end{array}$ & & & & \\
\hline Agrpecsilv & $\begin{array}{l}\text { Forestal } \\
\text { (subsector) }\end{array}$ & 13 & 9 & 7 & 3 & 8 & 6 \\
\hline Pesca & Pesca & 1 & 11 & 9 & 1 & 7 & 1 \\
\hline Minería & & 3 & 4 & 19 & 4 & 2 & 2 \\
\hline Alimen & Alimentos & 16 & 16 & 8 & 12 & 16 & 16 \\
\hline Textil & & 20 & 18 & 18 & 11 & 3 & 17 \\
\hline Madera & $\begin{array}{l}\text { Maderero } \\
\text { (subsector) }\end{array}$ & 14 & 17 & 4 & 13 & 12 & 14 \\
\hline Papel & & 12 & 15 & 11 & 16 & 19 & 19 \\
\hline Química & $\begin{array}{l}\text { Petroquímico- } \\
\text { Plástico }\end{array}$ & 4 & 3 & 10 & 14 & 13 & 7 \\
\hline Nomet & & 5 & 12 & 17 & 10 & 14 & 13 \\
\hline Metbas & & 7 & 6 & 13 & 9 & 10 & 8 \\
\hline Metmec & Metalmecánico & 8 & 2 & 16 & 7 & 5 & 4 \\
\hline Rindus & & 2 & 19 & 20 & 15 & 1 & 12 \\
\hline EGA & & 18 & 10 & 14 & 18 & 17 & 20 \\
\hline Constr & & 11 & 8 & 2 & 2 & 15 & 5 \\
\hline Comercio & & 6 & 5 & 1 & 5 & 18 & 3 \\
\hline Transp & $\begin{array}{l}\text { Transporte y } \\
\text { Logística }\end{array}$ & 9 & 7 & 6 & 6 & 20 & 9 \\
\hline Comun & & 10 & 14 & 15 & 8 & 4 & 10 \\
\hline Ssfin & & 17 & 1 & 5 & 20 & 9 & 11 \\
\hline Srvprop & & 19 & 13 & 3 & 19 & 11 & 15 \\
\hline Admpub & & 15 & 20 & 12 & 17 & 6 & 18 \\
\hline
\end{tabular}

Fuente: Elaboración propia.

En la última columna de la Tabla es expuesto el ranking global, el cual indica que los sectores con el mayor impacto positivo en el desarrollo económico de la región serían en orden decreciente: 'Pesca', 'Minería', 'Comercio, Restaurantes y Hoteles', 'Productos Metálicos, Maquinaria y Equipos', 'Construcción', 'Agropecuariosilvícola', 'Metálica Básica', 'Química, Petróleo, Caucho y Plástico', 'Transporte', 'Comunicaciones', 'Servicios Financieros y Empresariales', 'Resto de Industria', 'Fabricación de Productos No Metálicos', 'Madera y Muebles', 'Servicios Personales y Propiedad de Vivienda', 'Alimentos, Bebidas y Tabaco', 'Textil, Prendas de Vestir y Cuero', 'Administración Pública', 'Papel e Imprentas' y finalmente 'Electricidad, 
Gas y Agua'. Si se busca el desarrollo económico de la región con un énfasis más allá de lo netamente productivo, ponderando equitativamente cada criterio, los resultados apuntan a que los primeros sectores de esta lista debiesen ser potenciados por políticas de fomento. Cabe destacar que este tipo de análisis se centra en evaluar a los sectores en base al nivel de interrelación que presentan con el resto de los agentes económicos, independiente del tamaño de los sectores en el sistema económico regional bajo estudio.

Al centrar el análisis en los sectores priorizados por la ARIDP BIO BIO (de los ocho sectores escogidos consideramos los seis ligados a la SAM utilizada) concluimos que en general pertenecen al conjunto de los diez sectores de mayor importancia para el desarrollo económico de la región, donde el sector 'Forestal-Maderero' en conjunto ha sido incluido dentro de esta categoría pese a que el subsector 'Maderero' sólo ocupa la $14^{\mathrm{a}}$ posición. Otra excepción la constituye el sector 'Alimentos' que se ubica en el $16^{\circ}$ puesto. Alternativamente, desde una perspectiva más crítica podríamos decir que existen sectores en posiciones destacadas del ranking de desarrollo económico que no fueron priorizadas por la ARIDP BIO BIO como el sector 'Comercio, Restaurantes y Hoteles', 'Minería' y 'Construcción' que están dentro de los cinco sectores con mayor impacto del desarrollo económico.

Parte de la concordancia de nuestro ranking con los sectores de la ARIDP BIO BIO se explica porque la autoridad consideró entre otros criterios un análisis de encadenamientos para escoger los sectores priorizados, aunque utilizando la información desactualizada de la matriz insumo producto regional de 1996 y sin ponderar por los otros criterios de desarrollo económico propuestos en este estudio.

\section{CONCLUSIONES}

El trabajo desarrollado en este estudio involucra la construcción de una Matriz de Contabilidad Social Extendida Ambientalmente (ESAM), aplicando el método indirecto de construcción conocido como Cross Entropy para actualizar una Matriz de Contabilidad Social desde el año 1996 al 2006, e incorporándole posteriormente información ambiental relativa a las emisiones contaminantes generadas por los sectores productivos de la región.

La construcción de SAM regionales no es un tema nuevo en el país, pero previo a este trabajo no existía una SAM actualizada para la Región del Bío Bío. En este sentido, el trabajo constituye un aporte ya que contar con una SAM actualizada es relevante para una economía regional por sus amplias aplicaciones.

A partir de la nueva base de datos regional se genera un conjunto de indicadores basado en multiplicadores contables que permiten ordenar los sectores productivos de la Región del Bío Bío en función de su impacto sobre el desarrollo económico regional. Para esto, el análisis pondera los impactos sobre el encadenamiento en la producción regional, pobreza, distribución del ingreso y sobre el nivel de emisiones contaminantes que los sectores generarían ante un escenario de incremento de su demanda final. Como aplicación práctica se evaluó si los sectores escogidos por la Agencia Regional de Innovación y Desarrollo Productivo Región del Bío Bío (ARIDP 
$\mathrm{BIO} \mathrm{BIO})$, realmente son los sectores que al ser potenciados traen los mayores beneficios para el desarrollo económico de la región.

Como principales resultados a partir de la ESAM para la Región del Bío Bío tenemos que al ponderar equitativamente a los cuatro ámbitos estudiados, los sectores más beneficiosos para la región serían: 'Pesca', 'Minería', 'Comercio, Restaurantes y Hoteles', 'Productos Metálicos, Maquinaria y Equipos', 'Construcción', 'Agropecuariosilvícola', 'Metálica Básica', 'Química, Petróleo, Caucho y Plástico', 'Transporte' y 'Comunicaciones'. En cuanto a los sectores priorizados por la ARIDP BIO BIO, los resultados apuntaron a que en la mayoría de los casos éstos se encontrarían dentro del conjunto de sectores más relevantes para la región. Sin embargo, existen sectores en posiciones destacadas del ranking de desarrollo económico que no fueron priorizados como 'Minería', 'Construcción' y 'Comercio, Restaurantes y Hoteles' (este último no fue priorizado en su totalidad pero sí fue seleccionado por la Agencia el subsector denominado 'Turismo'). En el caso del sector priorizado 'Forestal-Maderero' según nuestros indicadores el subsector 'Maderero' tiene un bajo impacto en el desarrollo económico. Además se concluye que los sectores que más ingreso le reportarían al quintil más pobre son 'Comercio', 'Servicios Personales y Propiedad de Vivienda' y 'Construcción', ninguno de los cuales fue seleccionado por la ARIDP BIO BIO. Mientras por otro lado, algunos sectores priorizados tienen un fuerte impacto en la emisión de al menos uno de los contaminantes.

Por último, la precisión de la estimación de la ESAM para la Región del Bío Bío no podrá ser analizada mientras no se conozcan valores actualizados oficiales para la Matriz Insumo-Producto de esta región (elaborada ya sea por el INE, Banco Central u otro organismo). En este sentido, aún queda bastante trabajo por hacer de parte de las autoridades para contar con este tipo de información, tal como lo demuestra el hecho de que la fuente regional oficial más actualizada que existe del consumo intermedio regional sea la disponible en la Matriz Insumo-Producto del año 1996. Contar con estadísticas regionales de calidad con una frecuencia mayor a la actual debiese ser algo prioritario, pues en el caso de las SAM, aun si no se contase con toda la información requerida, al menos esto permitiría reducir el número de estimaciones usadas en la aplicación de los métodos indirectos de construcción como el Cross Entropy.

\section{REFERENCIAS}

ACEITUNO, G. (2008). "Consistencia Transversal en Cuentas Nacionales: Métodos de Reconciliación a través de Técnicas de Optimización”, Estudios Económicos Estadísticos Banco Central de Chile $\mathrm{N}^{\circ} 66$.

AROCA, P. y M. ATIENZA (2008). "La conmutación regional en Chile y su impacto en la Región de Antofagasta", Revista Eure 34 (102), pp. 97-120.

BARTELMUS, P., C. STAHMER y J. VAN TONGEREN (1991). "Integrated Environmental and Economic Accounting: Framework for a SNA Satellite System”, Review of Income and Wealth 37 (2), pp. 111-148.

CASANELLO, M. y M. HANNE (2004). "Metodología para la construcción de matrices de contabilidad social regionales para Chile y una aplicación para la Región de Antofagasta y la comuna de Valdivia", Universidad de Chile, Facultad de Economía y Negocios, Departamento de Economía.

CIASCHINI, M. y C. SOCCI (2007). "Final demand impact on output: A macro multiplier approach", Journal of Policy Modeling 29 (1), pp. 115-132. 
DEFOURNY, J. y E. THORBECKE (1984). "Structural Path Analysis and Multiplier Decomposition within a Social Accounting Matrix Framework”, The Economic Journal 94 (373), pp. 111-136.

GARCIA, A. y M. CASTILLO (1984). "Una Matriz de Contabilidad Social para Chile, 1977, Aspectos Metodológicos y Resultados", Monografías sobre empleo.

GILLESPIE, G., P. G. McGREGOR, J. K. SWALES y Y. P. YIN (2001). "The displacement and multiplier effects of regional selective assistance: a computable general equilibrium analysis", Regional Studies 35, pp. 125-139.

KEUNING, S. y W. DE RUIJTER (1988). "Guidelines to the Construction of a Social Accounting Matrix", Review of Income and Wealth 34 (1), pp. 71-100.

KEUNING, S. y J. TIMMERMAN (1995). "An information system for economic, environmental and social statistic: integrating environmental data into the SESAME", en Conference on Natural Resource and Environmental Accounting, Washington, DC.

KULLBACK, S. y R. LEIBLER (1951). "On information and sufficiency", Annals of Mathematical Statistics 22 (1), pp. 79-86.

LAGOS, C., C. DE MIGUEL, S. MILLER y R. O’RYAN (2003). "Una Matriz de Contabilidad Social para Chile 1996: Diseño y Metodología”, Instituto de Asuntos Públicos, Universidad de Chile.

LLOP, M. y A. MANRESA (2004). "Income Distribution in a Regional Economy: A SAM Model", Centro de Estudios Andaluces Economic Working Papers E2003/03.

MARDONES, C. (2009). "Estrategia de Desarrollo Región del Bío Bío ¿Escogimos los Sectores Correctos?”, Encuentro Anual de la Sociedad de Economía de Chile, Antofagasta 2009.

MIDEPLAN (2005). "Aproximación a las Economías Regionales con base en las Matrices de Insumo Producto Regionales del Año 1996". Santiago de Chile.

MORILLA, C. y G. LLANES (2004). "Matriz de contabilidad social y medioambiental: Aplicación a las emisiones de gases efecto invernadero de la Economía Española del año 2000”, Estudios sobre la Economía Española 181.

PARTRIDGE, M. y D. RICKMAN (2010). "Computable General Equilibrium (CGE) Modelling for Regional Economic Development Analysis", Regional Studies 44 (10), pp. 1311-1328.

PYATT, G. y A. ROE (1977). "Social Accounting for Development Planning with special reference to Sri Lanka”, Cambridge, Cambridge University Press.

PYATT, G. y J. ROUND (1985). "Social Accounting Matrices: A Basis for Planning”, Washington DC: The World Bank.

PYATT, G. y E. THORBECKE (1976). Planning Techniques for a Better Future, ILO, Geneva.

REINERT, K. y D.W. ROLAND-HOLST (1997). "Social Accounting Matrices", en J. Francois y K. Reinert, eds., Applied Methods for Trade Policy Analysis: A Handbook, Cambridge, Cambridge University Press.

RICKMAN, D. (1992). "Estimating the impacts of regional business assistance programs: alternative closures in a regional model", Papers in Regional Science 71, pp. 421-435.

ROBINSON, S. (1989). "Multisectoral Models", en H. Chenery y T. Srinivasan, eds., Handbook of Development Economics, Elsevier Science Publishers.

ROBINSON, S., A. CATTANEO y M. EL-SAID (2001). "Updating and Estimating a Social Accounting Matrix Using Cross Entropy Methods”, Economic Systems Research 13 (1), pp. 47-64.

ROBINSON, S. y M. EL-SAID (2000). "Gams Code For Estimating A Social Accounting Matrix (SAM) Using Cross Entropy (CE) Methods", Trade and Macroeconomics Division, International Food Policy Research Institute.

ROJAS, C. (2009). "Matriz de contabilidad social y análisis de multiplicadores contables para la Región Metropolitana de Santiago", Santiago, Chile: Universidad de Chile.

ROUND, J. (2003). "Social Accounting Matrices and SAM-based Multiplier Analysis", en F. Bourguignon y L. Pereira da Silva, eds., Techniques and Tools for Evaluating the Poverty Impact of Economic Policies, World Bank and Oxford University Press.

SHANNON, C. (1948). "A mathematical theory of communication", Bell System Technical Journal 27 (4), pp. 379-423.

STONE, R. (1954). "Input-Output and the Social Accounts", en The Structural Interdependence of the Economy, New York: Wiley and Sons, Inc.

URIEL, E., J. FERRI y M. MOLTO (2005). "Estimation of an Extended SAM with household production for Spain 1995”, Economic Systems Research 17 (3), pp. 255-278. 
VANOLI, A. (2005). A history of national accounting, IOS Press, Amsterdam.

VENEGAS, J. (1995). "Matriz de Cuentas Sociales 1986: Una SAM para Chile", Serie de Estudios Económicos Banco Central de Chile 39.

VOS, R. y N. JONG (2003). "Trade Liberalization and poverty in Ecuador: a CGE macro-microsimulation analysis", Economic Systems Research 15 (2), pp. 211-232.

XIE, J. (1995). "Environmental Policy Analysis: A Computable General Equilibrium Model for China", Ph.D. Dissertation, Cornell University.

XIE, J. (2000). "An Environmentally Extended Social Accounting Matrix," Environmental \& Resource Economics 16 (4), pp. 391-406. 


\section{APENDICE A}

Para efectos de presentación, el nombre de las cuentas fue en muchos casos abreviado, por lo que en primer lugar se incluye el Tabla A1 con el significado de estas abreviaciones. Luego, se presenta una versión agregada de la ESAMR-2006 (véase Tabla A2).

\section{TABLA A1}

ABREVIATURAS USADAS EN LA ESAMR-2006

\begin{tabular}{|c|c|c|}
\hline Cuenta macro & $\begin{array}{l}\text { Abreviación usada } \\
\text { en cuentas micro }\end{array}$ & Significado \\
\hline \multirow{3}{*}{ Recursos Naturales (rr.nn.) } & agrpecsilv & Agropecuario-silvícola \\
\hline & pesca & Pesca \\
\hline & miner & Minería \\
\hline \multirow{10}{*}{ Industria } & alimen & Alimentos, Bebidas y Tabaco \\
\hline & textil & Textil, Prendas de Vestir y Cuero \\
\hline & mader & Madera y Muebles \\
\hline & papel & Papel e Imprentas \\
\hline & quimic & Química, Petróleo, Caucho y Plástico \\
\hline & nomet & Fab. de Productos Minerales No Metálicos \\
\hline & metbas & Metálica Básica \\
\hline & metmec & Productos Metálicos, Maquinaria y Equipos \\
\hline & rindus & Resto Industria \\
\hline & ega & Electricidad, Gas y Agua \\
\hline Construcción & constr & Construcción \\
\hline Comercio & comer & Comercio, Restaurantes y Hoteles \\
\hline \multirow{5}{*}{ Servicios } & transp & Transporte \\
\hline & comun & Comunicaciones \\
\hline & ssfin & Servicios Financieros y Empresariales \\
\hline & srvprop & Servicios Personales y Propiedad de Vivienda \\
\hline & admpub & Administración Pública \\
\hline \multirow{3}{*}{ Factor Trabajo } & nocalif & Personal No Calificado \\
\hline & semicalif & Personal Semicalificado \\
\hline & calif & Personal Calificado \\
\hline \multirow{6}{*}{ Instituciones } & $\mathrm{q} 1$ & Quintil 1 \\
\hline & $\mathrm{q} 2$ & Quintil 2 \\
\hline & $\mathrm{q} 3$ & Quintil 3 \\
\hline & $\mathrm{q} 4$ & Quintil 4 \\
\hline & q5 & Quintil 5 \\
\hline & gov & Gobierno \\
\hline
\end{tabular}


Tabla A-1 (continuación)

\begin{tabular}{|lll|}
\hline \multirow{2}{*}{ Cuenta macro } & $\begin{array}{c}\text { Abreviación usada } \\
\text { en cuentas micro }\end{array}$ & \multicolumn{1}{c|}{ Significado } \\
\hline \multirow{3}{*}{ Impuestos } & instax & Impuesto directo a la renta \\
& acttax & Impuestos indirectos a actividades \\
& vatax & Impuesto al valor agregado \\
& imptax & Aranceles de importación \\
\hline \multirow{2}{*}{ Exterior } & row & Resto del Mundo \\
& roc & Resto de Chile \\
\hline Ahorro/Inversión & S-I & Ahorro/Inversión \\
& dstk & Variación de existencias \\
\hline
\end{tabular}

Fuente: Elaboración propia. 


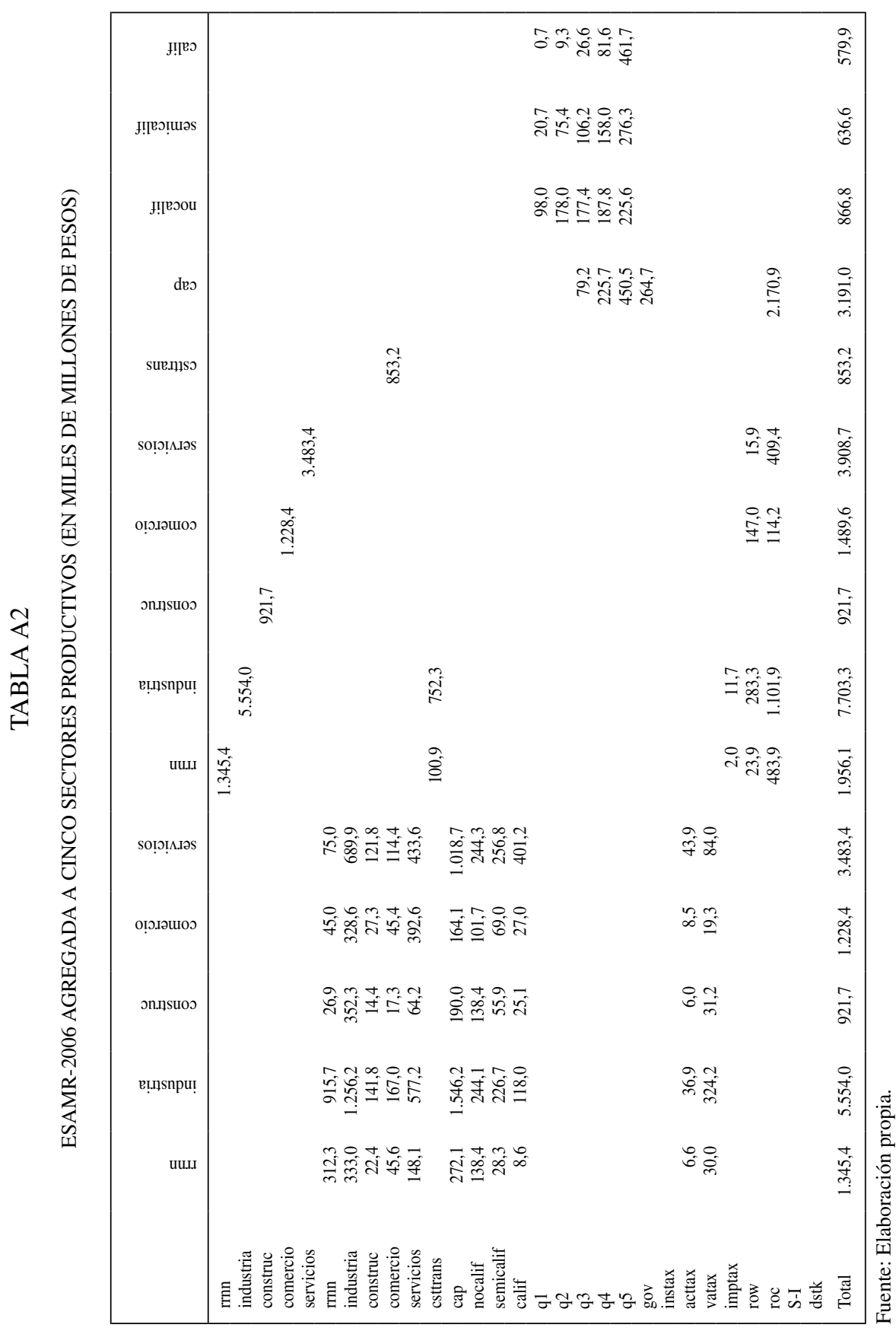




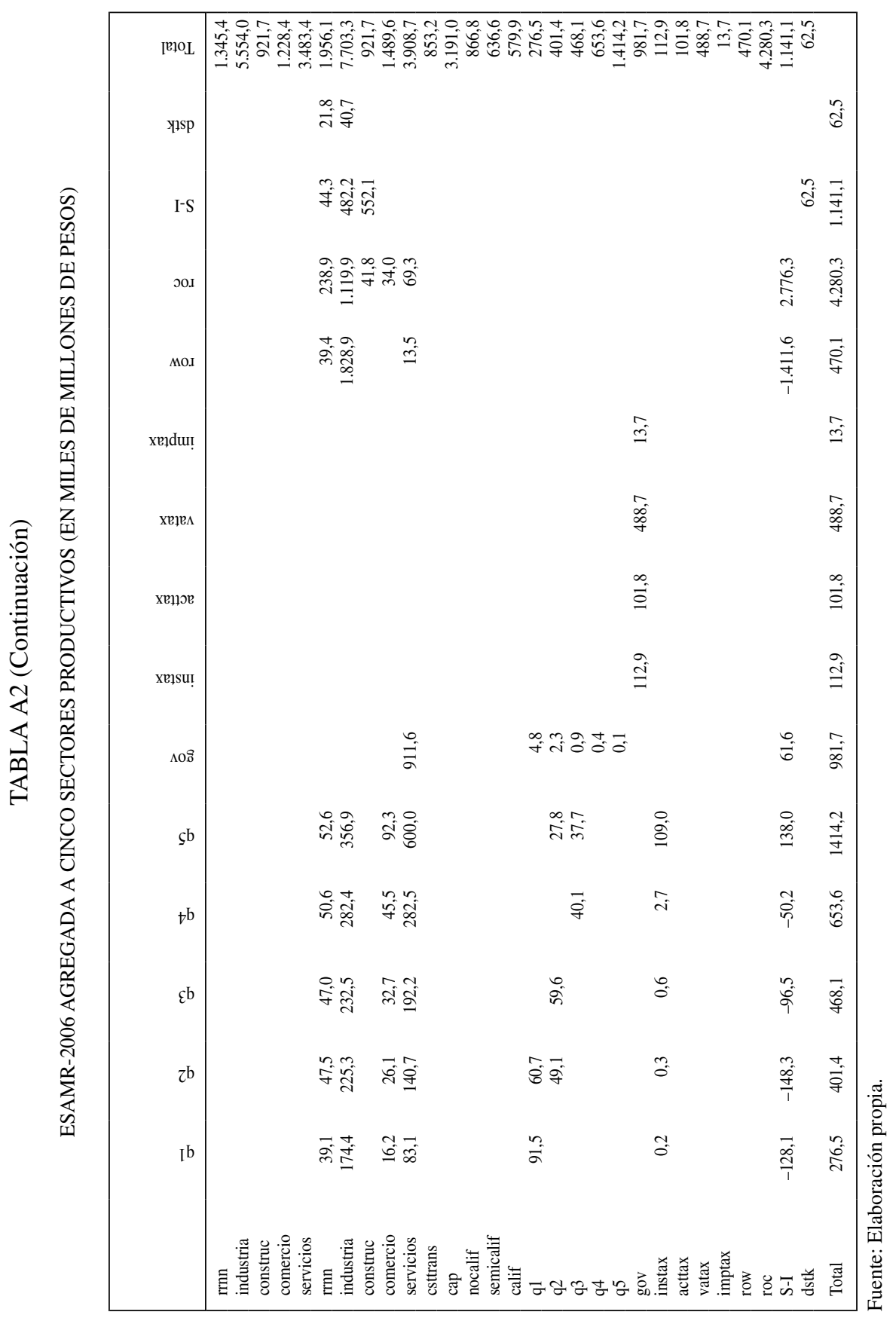


TABLA A2 (Continuación)

ESAMR-2006 AGREGADA A CINCO SECTORES PRODUCTIVOS (EN TONELADAS DE CONTAMINANTES)

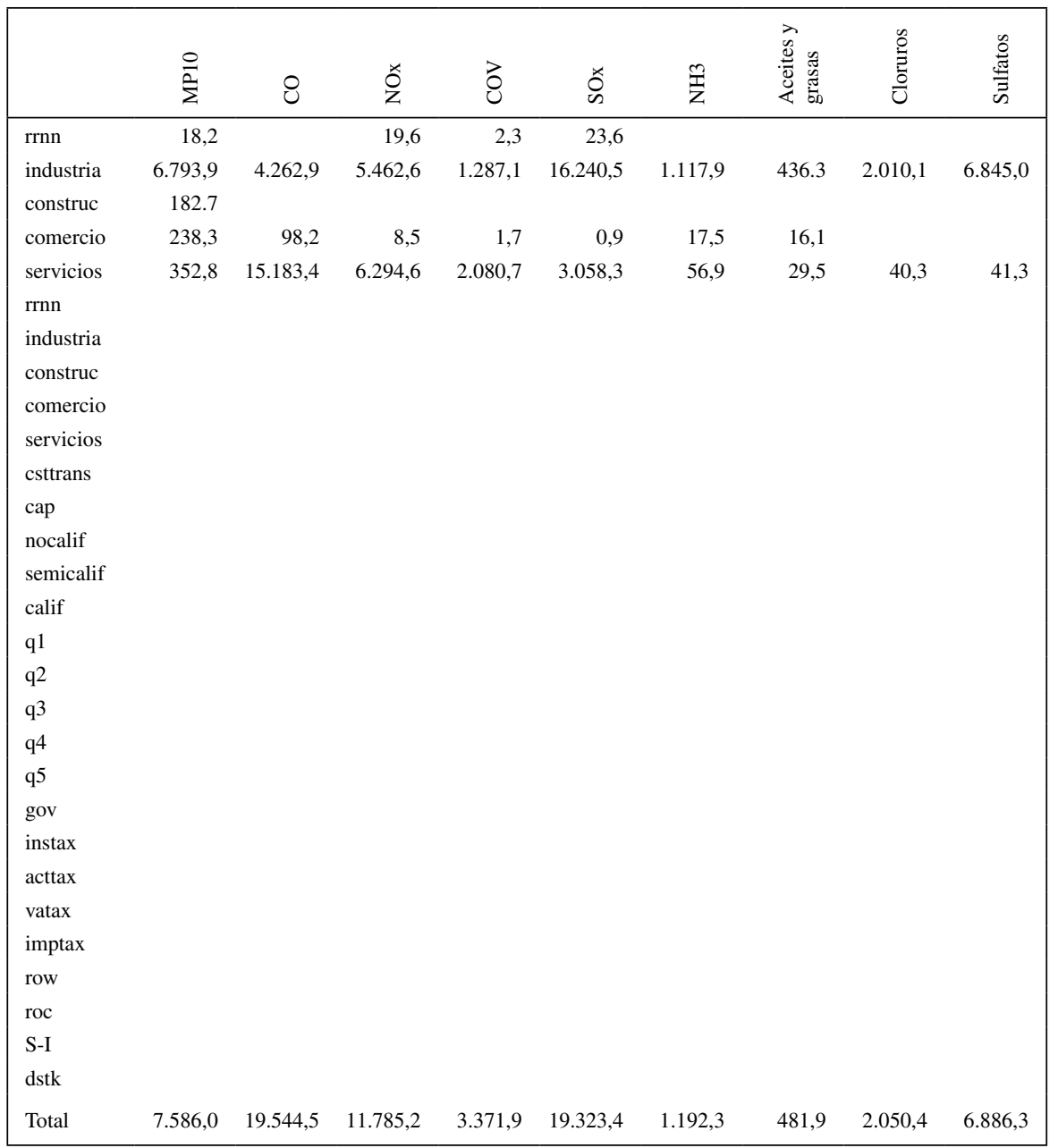

Fuente: Elaboración propia. 


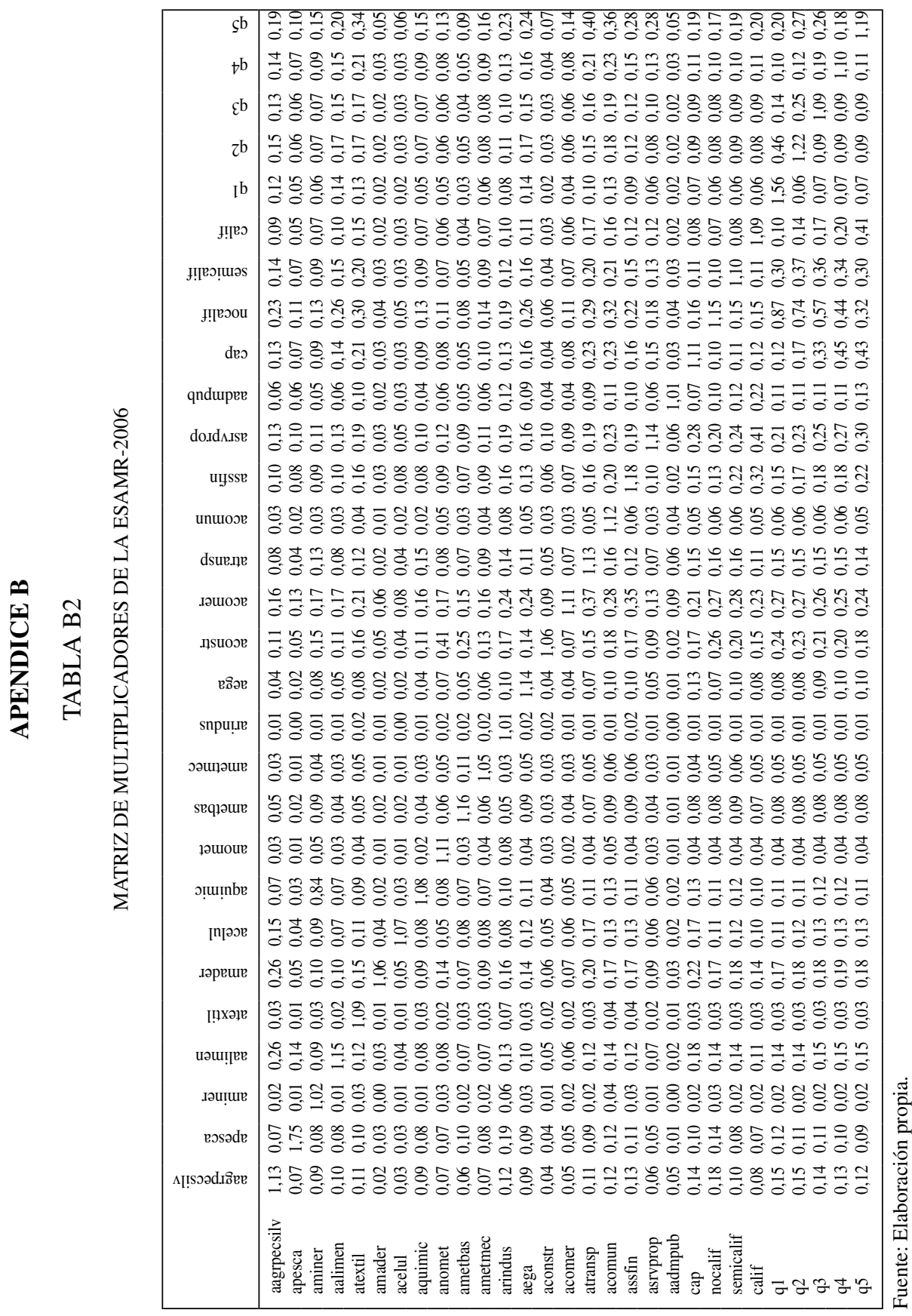




\section{APENDICE C}

En este Apéndice se indica la forma en que se generó el conjunto de restricciones potenciales definido en la aplicación del método Cross Entropy.

Para empezar, se obtuvieron tres vectores: uno con el gasto total en compras intermedias de los distintos sectores (vector $V$ ), otro con el valor agregado en su producción (vector $V A$ ), y el último, con la producción bruta de cada sector (vector $Y$ ). Respecto al último de estos vectores, es pertinente señalar que la medida utilizada a lo largo de este estudio para la producción bruta de un sector $j$ cualquiera es igual a:

$$
y_{j}=v_{j}+v a_{j}+i v a_{j}
$$

Donde $i v a_{\mathrm{j}}$ es igual al impuesto al valor agregado pagado por el sector $j$, y donde la definición de valor agregado empleada equivale a:

$v a_{j}=$ Remuneraciones $_{j}+$ Exc. Bruto de Expl $. j_{.}+$Impuestos indirectos a actividad $_{j}$

Para todos los sectores, salvo aquéllos de las industrias manufactureras ('Alimentos, Bebidas y Tabaco', 'Textil, Prendas de Vestir y Cuero', 'Madera y Muebles', 'Papel e Imprentas', 'Química, Petróleo, Caucho y Plástico', 'Fabricación de Productos Minerales No Metálicos', 'Metálica Básica', 'Productos Metálicos, Maquinaria y Equipos' y 'Resto Industria'), los elementos $v_{j}$ del vector $V$ fueron estimados suponiendo constante la razón ' $v_{\mathrm{j}} / v a_{\mathrm{j}}$ ' entre los años 1996 y 2006, con los valores del año 1996 obtenidos de la SAM del mismo año. El uso de este supuesto se basa en asumir una estructura de costos y función de producción constante, lo que si bien en el corto plazo pudiera ser razonable, en el plazo considerado de 10 años, es cuestionable. Pese a lo anterior, la poca disponibilidad de datos no dejó otra opción.

El vector de valor agregado (VA) actualizado al 2006, por su parte, fue obtenido en base a estadísticas del Banco Central del PIB regional por clase de actividad económica para el periodo 2003-2006. Estos valores se encontraban en moneda constante del 2003, por lo que la obtención de valores en pesos del 2006 fue hecha usando la medida de inflación dada por la razón del Indice de Precios al Consumidor (IPC) de los años 2003 y 2006.

Para el caso de las industrias manufactureras, tanto los elementos $v_{\mathrm{j}}$ como $v a_{\mathrm{j}}$ de estos sectores fueron obtenidos en base a la Encuesta Nacional Industrial Anual (ENIA) desarrollada por el INE. La encuesta contenía información del consumo intermedio y valor agregado regional (precios corrientes), para 75 subsectores del sector manufacturero. Luego, la utilización de estos datos requirió la asignación de estos 75 sectores a los 9 (sectores industriales) considerados en el estudio.

Dentro de las fuentes de información empleadas, se consideró a las provenientes del Banco Central como las más confiables. Por esto, al disponerse del valor agregado 
contabilizado por este organismo para el sector industrial (agregado), se optó por ajustar los datos de la ENIA en base a la razón entre ambos totales. Asumiendo que esta diferencia pudiera darse también en torno al consumo intermedio, éste fue igualmente ajustado en base a la misma razón anterior. ${ }^{11}$

Disponiéndose tanto del valor agregado como del gasto en consumo intermedio de todos los sectores, el único elemento faltante para la obtención del vector $Y$ (vector de producción bruta) era el vector de impuesto al valor agregado (IVA). Asumiendo que la relación ' $i v a_{\mathrm{j}} / v a_{\mathrm{j}}$ ' regional era similar a la nacional, se utilizó la razón obtenida de la Matriz Insumo-Producto nacional del 2003, la cual era la fuente más actualizada. Cabe señalar que esta matriz se encontraba desagregada a 73 sectores, por lo que la obtención de esta razón requirió la previa agregación de estos sectores a los 20 considerados del análisis. Así, el IVA cancelado por cada sector en el 2006 fue calculado en base a esta razón y a los valores de valor agregado obtenidos anteriormente. Una vez realizado lo anterior, el vector $Y$ fue calculado como la suma de los vectores $V, V A$ e IVA.

En cuanto a las demás restricciones potenciales, los valores de éstas surgieron a partir de las siguientes estimaciones.

- Aranceles: obtenidos aplicando una tasa arancelaria nacional por tipo de producto sobre las importaciones desde el resto del mundo. La tasa, por su parte, fue aproximada como la razón entre los aranceles e importaciones (según el sector correspondiente) incluidos en la MIP nacional del 2003.

- Pago al factor trabajo: los valores fueron obtenidos en base a la razón ' $l a b_{j} / v a_{\mathrm{j}}$ ' regional del '96, asumiendo constante esta relación. ${ }^{12}$

- Pago al factor capital: obtenido de forma análoga al caso anterior, pero en base a la razón ' $c a p_{j} / v a_{j}$ '. 13

- Pago al factor trabajo que va como ingreso a hogares regionales: basándose en información de Aroca y Atienza (2008), respecto a que la razón nacional tanto de migración como de conmutación laboral interregional son inferiores al 2,6\% de la población activa (estadísticas del Censo del 2002), se optó por despreciar estos factores, lo que se traduce en asumir que este ingreso equivale al pago total al factor trabajo hecho por las actividades productivas regionales.

- Consumo de hogares de la Octava Región: el consumo total se obtuvo en base a la razón 'Consumo Total / VA Total' nacional del 2006 (a precios corrientes) y el VA regional del mismo año. ${ }^{14}$ Por su parte, la desagregación de este consumo total entre los distintos sectores fue hecha en base a la VI Encuesta de Presupuestos Familiares (EPF), utilizando la proporción del gasto promedio mensual de los hogares destinada a los distintos sectores. Para esto, fue necesario asociar cada uno de los 50 sectores considerados por esta encuesta a los 20 de este estudio.

11 No fue posible ajustar el consumo intermedio en base a una razón de consumo intermedio, pues el Banco Central no genera estadísticas anuales regionales en torno a esto, por lo que no se disponía del dato para el 2006.

12 Con ' $l a b$ ' igual al pago al factor trabajo.

13 Con 'cap' igual al pago al factor capital.

14 Ajustado por inflación en base a los IPC promedio de los años 2003 y 2006. 
- Ingreso total de los hogares: conocido el consumo total (o gasto total) de los hogares, la obtención de este valor requirió el uso de éste y de la razón 'Ingreso Promedio / Gasto Promedio' mensual por hogar, obtenida de la EPF. Si bien es posible obtener una estimación del ingreso total empleando la CASEN 2006, se optó por finalmente no utilizarla.

- Impuestos indirectos a actividades: obtenidos como la diferencia entre el VA de los sectores y el pago a los factores capital y trabajo.

- Impuestos directos: fue calculado como el 4,16\% del ingreso total de los hogares, cifra promedio obtenida en base a información del Servicio de Impuestos Internos (SII).

- Gasto público regional: el consumo total del gobierno fue calculado en base a la razón 'Gasto Público / Población' nacional y la población regional proyectada por el INE para el 2006. La desagregación de este gasto entre los sectores fue hecha usando las mismas proporciones de la SAM regional del año 1996.

- Inversión Pública Regional: obtenida de la Serie Regionalizada de Inversión Pública Efectiva publicada por el MIDEPLAN.

- Subsidio del gobierno a hogares de la región: este valor se obtuvo a partir de la Encuesta CASEN del año 2006.

- Pago al factor capital retribuido al gobierno: ${ }^{15}$ calculado como la diferencia entre el gasto total ${ }^{16}$ del gobierno (suma de los tres puntos anteriores) y el ingreso total de éste proveniente de los impuestos.

- Pago al factor capital retribuido a hogares de la región: disponiéndose del ingreso total de los hogares regionales, este valor se obtuvo como la diferencia entre éste y las demás fuentes de ingreso (subsidios y remuneraciones).

- Pago al factor capital que va como ingreso a hogares de otras regiones: calculado como la diferencia entre el ingreso total de esta cuenta y los otros gastos de ella.

- Exportaciones al resto del mundo: información obtenida del Servicio Nacional de Aduanas. Los valores se encontraban expresados en dólares, por lo que la conversión a pesos del 2006 fue hecha en base al promedio del tipo de cambio diario observado durante ese año.

- Inversión total en bienes y servicios asociados a los sectores de la región: la inversión total fue calculada en base a la razón 'Inversión Total / VA Total' nacional y el VA regional del 2006 a precios corrientes de ese año. La desagregación de esta inversión entre los sectores fue hecha en base a la SAM regional del '96.

- En cuanto al resto de las transacciones, se optó por permitir que fuera el método Cross Entropy el que las generara, en base a la estructura de la SAM de 1996 y al conjunto de restricciones finalmente implementadas.

15 Corresponde al pago a este factor hecho por empresas públicas.

16 Que por las características de la SAM equivale al ingreso total. 
